\title{
Optical spectroscopy of radioactive atoms
}

\author{
H. Henry Stroke
}

Published online: 27 March 2007

(C) Springer Science + Business Media B.V. 2007

\begin{abstract}
An epitome of optical methods used in atomic spectroscopy of radioactive atoms is presented. The overview addresses a number of results in atomic structure and hyperfine structure, and the implications in the study of electric and magnetic properties of nuclei. An aperçu is given of the concomitant development of the experimental methods, from simple optical techniques to laser spectroscopy, and from use of "off-line" experiments to ones using ISOLDE-type facilities.
\end{abstract}

Keywords Radioactive atom spectroscopy • Isotope shifts • Hfs • Laser spectroscopy $\cdot$ Nuclear multipole moments

\section{Introduction}

A brief historical accounting, paralleling the presentation at the VII International Workshop Laser 2006 in Poznan, Poland, is made of the spectroscopy of radioactive atoms, from its beginning to the present day. It is forcibly sketchy, relying largely on personal experience and acquaintance, certainly not exhaustive, and, in the process, it omits a great number of important contributions of colleagues, for which I apologize. Nonetheless, it hopefully presents a picture - highlights - which should give an appreciation of the richness of the physics results obtained and the effort of many, over nearly a century, in developing more and more sensitive and precise

H. H. Stroke (凶)

Department of Physics, New York University, 4 Washington Place,

New York, NY 10003, USA

e-mail: henry.stroke@nyu.edu

H. H. Stroke

CERN, 1211 Geneva 23, Switzerland 
Fig. 1 Jacques Pinard (courtesy of J.P.)

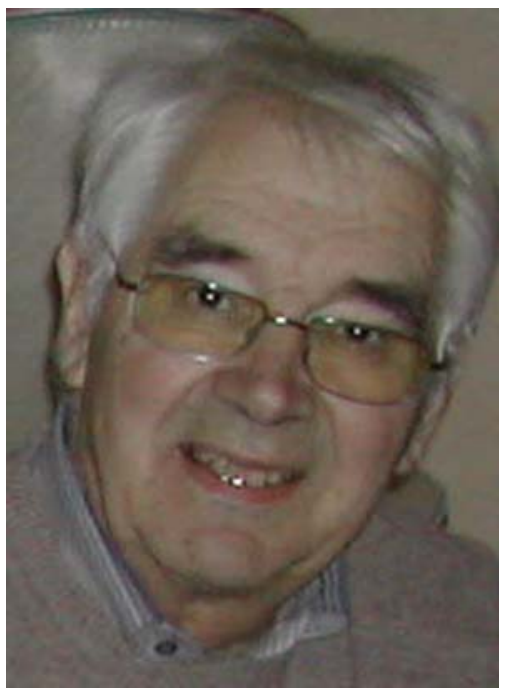

experimental techniques to reach the results. I give a sampling of the literature, from old to current, which should provide some indication of these efforts [1-12]. ${ }^{1}$ This presentation was aided substantially by a recent review that was made in collaboration with Jacques Pinard, Fig. 1, of the Laboratoire Aimé Cotton in Orsay [13].

As an introduction, the extension of "classical" spectroscopy to radioactive atoms is discussed. By classical we refer to the light sources or absorption cells, not to the methods: these were in fact extended radically from early dispersive grating and Fabry-Pérot techniques to Fourier spectroscopy [14-16], ${ }^{2}$ which allows an entire spectrum to be recorded with high resolution. The first radioactive atom spectroscopy is in fact that of ${ }^{209} \mathrm{Bi}$, in 1926, which has been found to be an $\alpha$ emitter only recently, with a half-life of $1.9 \times 10^{19}$ years [17]. In the early $1930 \mathrm{~s}$, less exotic radioisotopes, of radium and radon, were measured, and can be found listed in Charlotte Moore's Atomic Energy Levels III table.

I digress for an instant. In 2005 the world celebrated the annus mirabilis of Einstein's monumental papers. The decade, starting in the vicinity of 1925 , constituted for physics in general, and for a number of subjects of concern here, veritable anni mirabiles, both for theory and experiment. In 1924, Wolfgang Pauli [18] suggested the existence of a nuclear spin and hyperfine structure in the atomic

\footnotetext{
${ }^{1}$ Refs. [7, 8] include the recent application at the Jyväskylä IGISOL facility of the "cooler-buncher" technique, which will represent a crucial advance for radioactive atom spectroscopy.

${ }^{2}$ I recall that Fourier spectroscopy is based on the Michelson interferometer where the fringe visibility is measured as one of the mirrors is displaced. This actually gives the Fourier transform of the spectrum. Michelson constructed an analogue device to obtain the inverse transform; this appears to have discouraged further spectroscopic applications, until digital techniques were introduced (see references in $[15,16])$.
} 
Fig. 2 Sam Goudsmit (courtesy American Institute of Physics, Emilio Segrè Visual Archives)

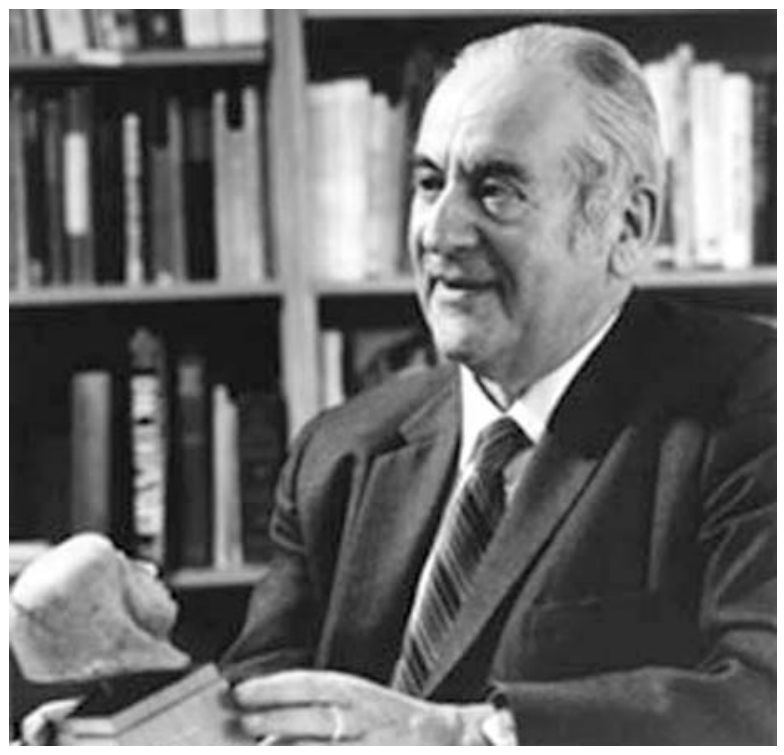

spectrum, then measured and interpreted for bismuth by Ernest Back and Sam Goudsmit (Fig. 2) [19, 20]. This would have been the accepted chronology, but for the correction brought to light recently by Takashi Inamura [21]. He brings to light the early studies of isotope shifts and hyperfine structure by Hantaro Nagaoka [22]. This, Inamura recounts, was recognized by Pauli but not in most of the atomic physics books with which we were educated. I note, however, that the "bible," Condon and Shortley [23], does give a reference to some of Nagaoka and co-workers isotope shift studies, as do Back and Goudsmit [19] to both this and earlier work. If I can be forgiven for a purely provincial outlook at New York University, I note the first theoretical paper on volume-dependent isotope shifts and the effect of a distributed nuclear charge distribution on atomic hyperfine structure by Jenny Rosenthal (Fig. 3) and Gregory Breit [24] (the so-called Breit-Rosenthal-CrawfordSchawlow correction), the calculation in 1931 by Herman Yagoda of the wavelengths of the resonance lines of the then not yet discovered unstable element, francium $[25,26]$, a subject to which I return, and, parenthetically, to finish with NYU, the first experiment on $\beta$-decay asymmetry, reported by Richard Cox, et al., though certainly unexplained at that time [27]! Before touching on some of the milestones, I call attention to the largely ignored paper by Victor Weisskopf [28] that later laid the foundation for level-crossing spectroscopy [29, 30] with fruitful applications in the study of hfs spectra of radioisotopes.

\section{Measurable quantities}

We divide the optical spectroscopies of radioisotopes into two broad areas of interest:

A. Atomic physics and interactions

B. Electron-nuclear interactions 
Fig. 3 Jenny Rosenthal Bramley at her 1929 NYU commencement: the first woman to earn a PhD in physics (courtesy NYU Alumni Today)

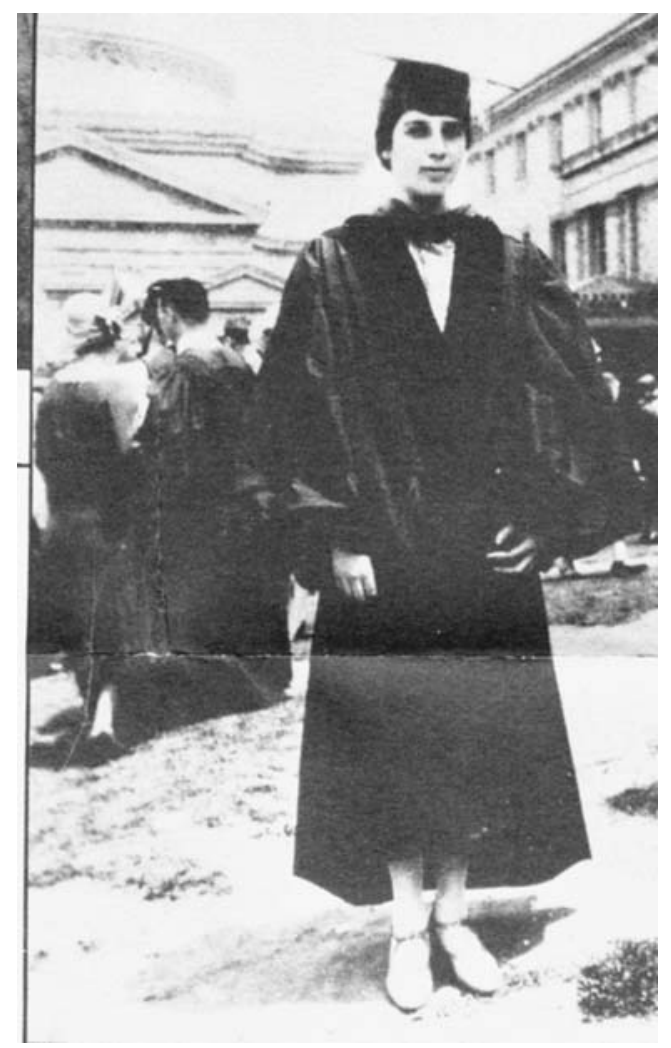

\subsection{Atomic physics}

\subsubsection{Actinides}

This series of radioisotopes, that corresponds to the lighter lanthanides, has been studied over many years (Jean-François Wyart, Jean Blaise) at the Laboratoire Aimé Cotton, long under the direction of Pierre Jacquinot (Fig. 4).

A number of other laboratories, Livermore, Argonne, Lund, Amsterdam, ..., have been actively engaged in the measurement and analysis of these spectra. The results are available on the site http://www.lac.u-psud.fr/LAC/data/database.htm. The classification of the atomic spectra relies on the Rydberg-Ritz principle, aided in the configuration assignments by observation of hfs and isotope shifts.

\subsubsection{Electron correlations}

Isotope shifts, discussed below, are the primary interest for nuclear structure studies. The extraction of this "volume effect" of the nuclear charge distribution is limited by mass-dependent effects. These depend in turn on the correlation of the motion of all the electrons [23] - and is thus a nuisance many-body problem, unfortunately important in light and middle- $A$ nuclei ( $A$, mass number). 
Fig. 4 Pierre Jacquinot (courtesy Laboratoire Aimé Cotton, Centre National de la Recherche Scientifique)

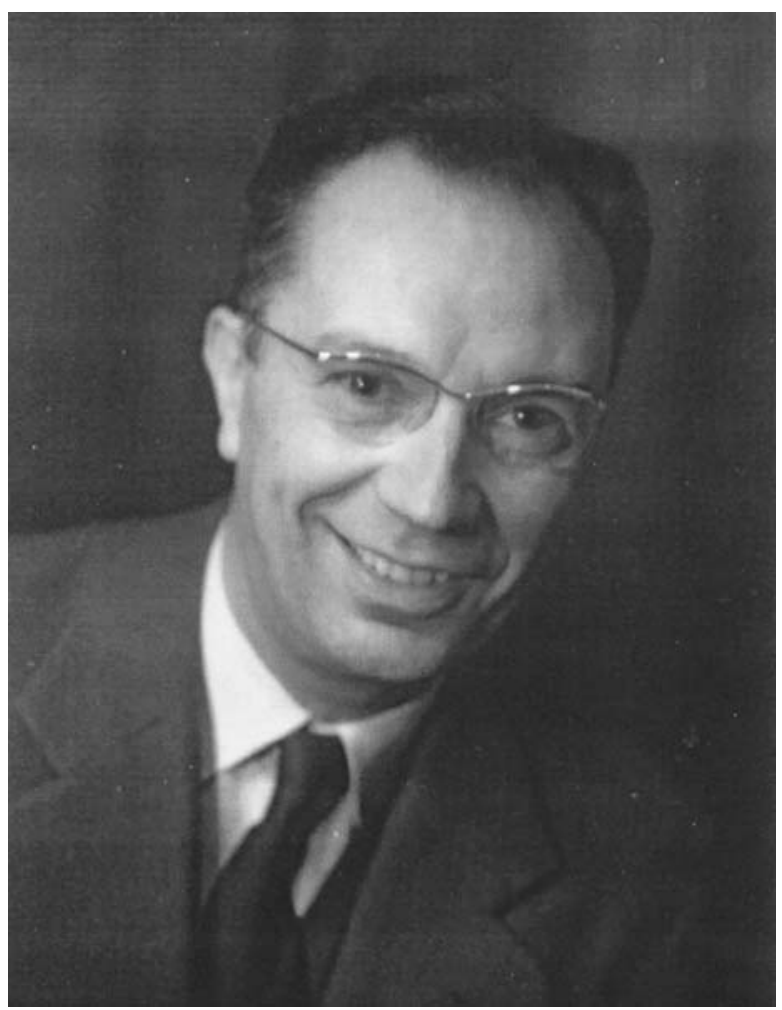

\subsubsection{Spectrum of francium}

Theoretical work by Yagoda $[25,26]$ on the spectrum of francium (called eka-cesium until its discovery in 1939 by Marguerite Perey [31]) (Section 1) relied on analyses of isoelectronic series. His predictions for the resonance lines proved to be closer to the experimental values $[32,33]$ than a number of more recent, sophisticated, calculated values. Although a finely tuned laser is not the best adapted tool for searching for an atomic transition over large wavelength ranges, experiments were successful for several resonance lines (Fig. 5).

\subsection{Electron-nuclear hyperfine interaction}

In his 1924 paper [18], Pauli encompasses a good part of the physics of hfs, including the effect of the extended charge distribution on the electron-nuclear interaction, i.e. the volume-dependent isotope effect. As mentioned in Section 1, in order to account for observed structures, he also introduced the existence of a nuclear spin, and notes that the electron-nuclear interaction is much smaller than the electronelectron interaction. Goudsmit and Robert Bacher [34] applied the resulting interval rule to fit measured spectra with this magnetic dipole interaction, but encountered difficulties: the electric quadrupole moment of the nucleus was not yet known. The inclusion of its interaction with the gradient of the atomic electric field at the position of the nucleus was calculated in the prize essay of Hendrik B.G. Casimir [35]. A 


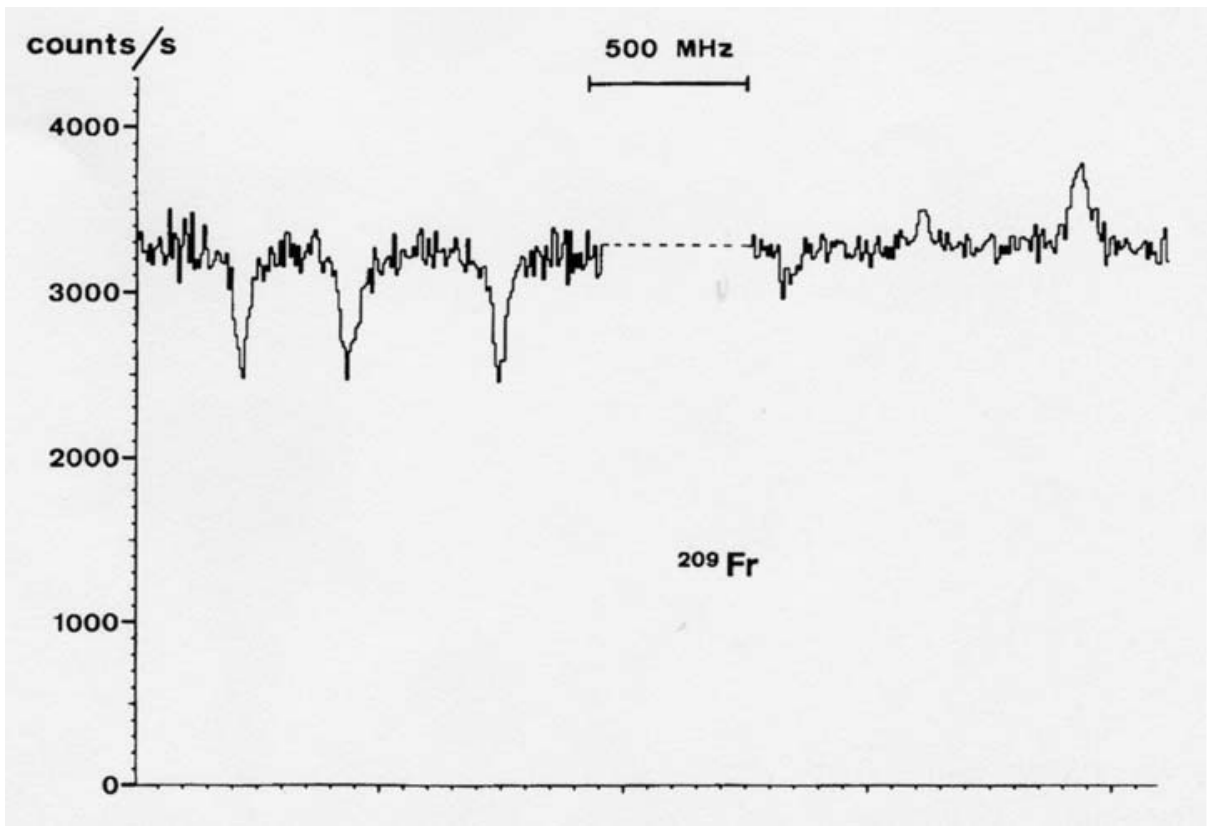

Fig. 5 Francium D2 line, from Ref. [33] (courtesy (CAmerican Physical Society, http://prola.aps.org/ abstract/PRA/v22/i6/p2732_1)

few years later, he also calculated [36] the next term in the electron-nuclear hfs interaction, caused by a possible nuclear octupole moment, which we detected a dozen years later [37].

\subsubsection{Isotope shifts}

In the preceding paragraph we discussed the multipole electron-nuclear interaction, but omitted the monopole term, the point Coulomb interaction. From the so-called "center-of-gravity" or "center-of-mass" theorem [38] we know that measurements of the hyperfine interactions (multipolarity $\mathrm{k}>0$ ) give no information about the center of gravity of the atomic level, determined by $\mathrm{k}=0$. The latter, on the other hand, is dependent on the spherically averaged value of the nuclear charge distribution, which, in turn, gives rise to the volume-dependent part of isotope shifts.

\subsubsection{Bohr-Weisskopf effect}

The magnetic counterpart of the isotope shift is the Bohr-Weisskopf (Fig. 6) effect, also known as the "hfs anomaly" [39]. It reflects the influence on the magnetic dipole hfs interaction of the spatially-distributed nuclear magnetization - both spin and orbit. For ordinary atoms the effect is observed via the comparison of isotopic ratios of hfs interaction constants with those of independently-measured nuclear $g$ factors. For atoms, this is a relatively small effect, for muonic atoms a large effect. But the enormously greater precision of atomic measurements makes the atomic spectroscopy much more sensitive to these nuclear effects. 
Fig. 6 Viki Weisskopf at the "Bohr-Weisskopf" atomic beam apparatus at ISOLDE (photo by author)

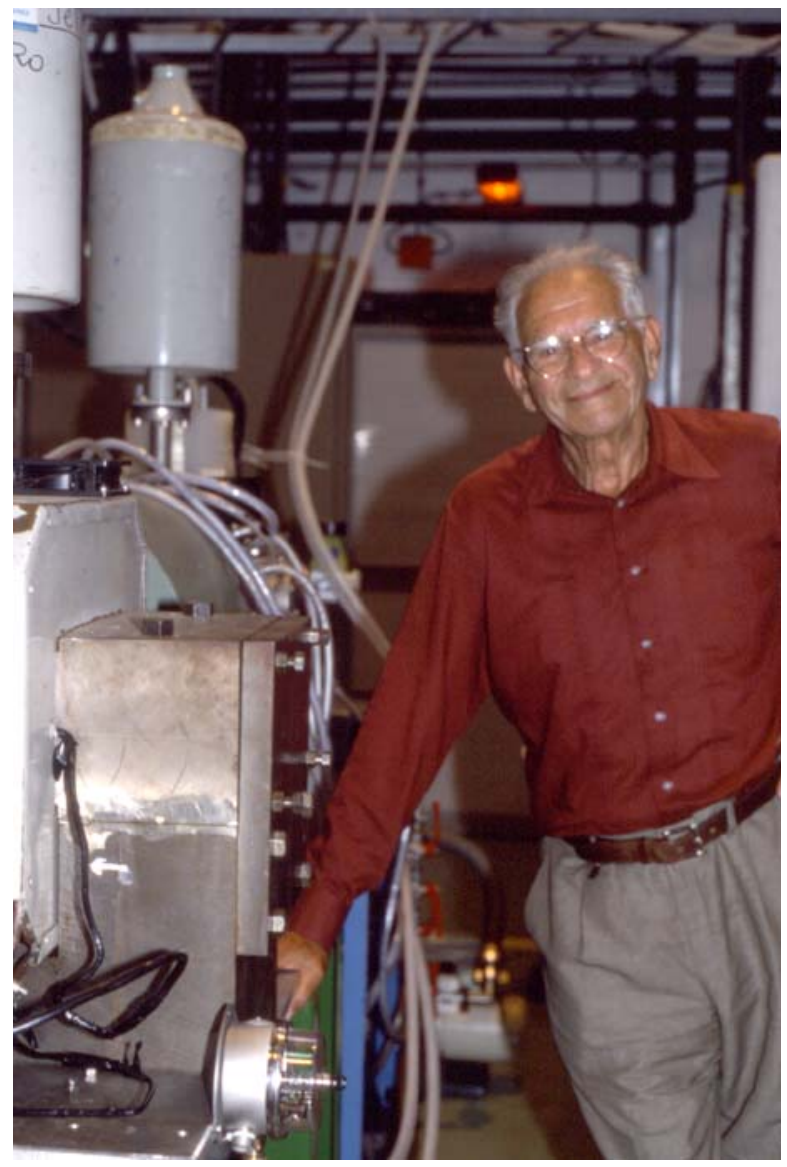

As a historical aside, we reproduce (Fig. 7) an introductory page of the first edition of the book of Hans Kopfermann [40].

What he neglects is in fact the Bohr-Weisskopf effect, which in many cases is only a fraction of one percent! One can forgive Kopfermann: his book was the first important compendium of this field, and he educated a whole generation of post-war physicists in Germany, many of whom became leaders in atomic physics in their own right. Kopfermann was also probably the first (or was among the first) German physicist to be invited after World War II to visit MIT, where he gave a very stimulating set of lectures on hfs. An English edition of his book was published later [41].

\section{Experimental methods}

\subsection{Atomic beam techniques}

The experiments of Otto Stern and Walther Gerlach led first to non-resonance atomic beam experiments, e.g. the "zero-moment" method for determining nuclear spins and hfs [42]: it consists, for alkali atoms, in determining in the Zeeman effect 


\section{B. Magnetische Wechselwirkungen}

In ganz entsprechender Weise läßt sich die magnetische Wechselwirkung zwischen Kern und Elektronenhülle durch ein Kernvektorpotential $\mathfrak{A}$ und die Stromdichte $\vec{\sigma}$ der Hüllenelektronen darstellen:

$$
V_{\text {magn }}=-\frac{1}{c} \int(\mathfrak{A}, \vec{\sigma}) d \tau .
$$

Man kann das Kernvektorpotential außerhalb des Kerns nach Potenzen von $\frac{1}{r}$ entwickeln, wobei das Vektorpotential in erster Näherung das eines magnetischen Kerndipols $\mathfrak{U}=\frac{\left[\overrightarrow{\mu_{I}}, \vec{r}\right]}{r^{3}}$ wird. Höhere magnetische Pole, welche sicher nur als kleine Störungen anzusehen sind, und für deren Existenz bisher keine experimentellen Anzeichen vorliegen, sollen unberücksichtigt bleiben. Dann wird:

$$
V_{m a g n}=-\frac{1}{c} \int \frac{\left(\left[\overrightarrow{\mu_{I}}, \vec{r}\right] \vec{\sigma}\right)}{r^{3}} d \tau=-\frac{1}{c} \int\left(\overrightarrow{\mu_{I}}, \frac{\vec{r}, \vec{\sigma}]}{r^{3}}\right) d \tau .
$$

Die Größe $\frac{1}{c} \int \frac{[\overrightarrow{r, \sigma]}}{r^{3}} d \tau$ stellt das Magnetfeld $\mathfrak{S}(0)$ dar, welches die Elektronen am Kernort erzeugen. Man erhält

$$
V_{\text {magn }}=-\left(\overrightarrow{\mu_{I}}, \mathfrak{G}(0)\right)=-\mu_{I} H(0) \cos \left(\overrightarrow{\mu_{I}}, \mathfrak{G}(0)\right) \text {. }
$$

Der Betrag $\delta H(0)$, der von dem innerhalb des Kernvolumens fließenden Teil der Elektronenstromdichte stammt, und der in $(1,5)$ nicht erfaßt ist, dürfte im allgemeinen unmerklich klein sein. Selbst bei den $s$-Elektronen der schweren Atome, welche dem Kern sehr nahekommen, kann die Vernachlässigung der magnetischen Wechselwirkung innerhalb des Kernrolumens nur einen Fehler von wenigen Prozenten in $(1,6)$ ausmachen.

Fig. 7 From the first edition of Kopfermann's book, Kernmomente [40]: he neglects the influence of the penetration of the electron inside the nuclear volume, which would lead to an error of only a few percent (courtesy of Akademische Verlagsgesellschaft, Berlin)

of the hfs the magnetic fields, $B$, for which the effective magnetic moment $\mu_{e f f}=$ $-\partial E / \partial B=0$ : such atoms are detected after traversing undeflected an inhomogeneous magnetic field. $E$ is the energy of a particular hyperfine level. An example for the 2.1-y ${ }^{134} \mathrm{Cs}[43]$ is shown in Fig. 8.

This was followed by the atomic (or molecular) beam magnetic resonance (ABMR) method of Rabi and co-workers [44], Fig. 9. Here, two inhomogeneous magnets, A and B, with their field gradients in opposite directions, were separated by a homogeneous magnetic field, $\mathrm{C}$, that also contained an rf loop. In the absence of rf transitions, the atoms are refocused on the detector by the B magnet. At the resonant frequency, atoms make transitions from positive to negative $\mu_{\text {eff }}$ or vice versa, producing a small decrease in detected atom intensity.

It was soon recognized by Jerrold Zacharias that having the magnetic-field gradients of the $\mathrm{A}$ and $\mathrm{B}$ magnets in the same direction would increase the signal-to-noise 
Fig. 8 The effective magnetic moment of the energy levels for the atom with electron angular momentum $J=\frac{1}{2}$ and nuclear angular momentum $I=4$. The solid circles represent the magnetic fields at which $\mu_{\text {eff }}$ vanishes. From [43] (courtesy (C)American Physical Society, http://prola.aps.org/abstract/ PR/v87/i4/p676_1)
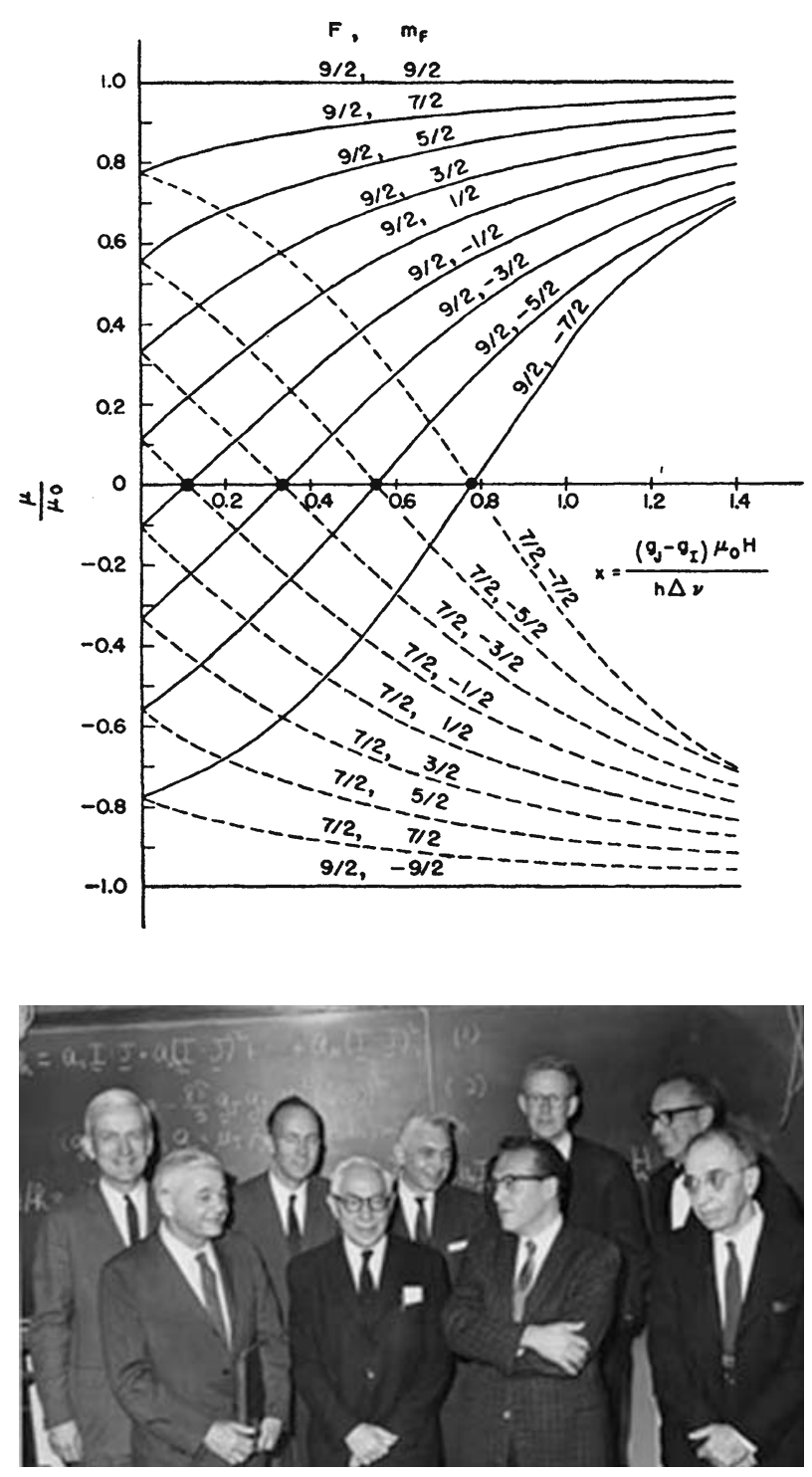

Fig. 9 Some of the key figures in ABMR and resonance physics. Back row: Norman Ramsey, Charles Townes, Vernon Hugues, Ed Purcell, William Nierenberg.

Front row: Jerrold Zacharias, I.I. Rabi, Julian Schwinger, Gregory Breit (courtesy American Institute of Physics, Emilio Segrè Visual Archives)

ratio considerably: one looks for a small signal on essentially zero background in the absence of resonance. This was implemented by Zacharias in the measurement [45] of $1.25 \times 10^{9}-\mathrm{y}^{40} \mathrm{~K}$, found in nature with an abundance of $0.0117 \%$. This was the beginning of radioactive ABMR work on alkali atoms at MIT [46], Fig. 10. (It ended in 1954 with a major radioactivity spill by the author!) I note that some $5 \times 10^{9} \mathrm{~Bq}$ of ${ }^{134} \mathrm{Cs}$ were typically loaded into the ABMR source (10 times less activity for a mixture of ${ }^{137,135} \mathrm{Cs}$ ).

A number of other laboratories around the world started work on radioactive ABMR. This was reviewed by William Nierenberg [1]. I only point out work on the first nuclear isomer, 2.9-h ${ }^{134 m} \mathrm{Cs}$, by Victor Cohen and Donald Gilbert at Brookhaven National Laboratory [47], and the development of a focussing ABMR, 


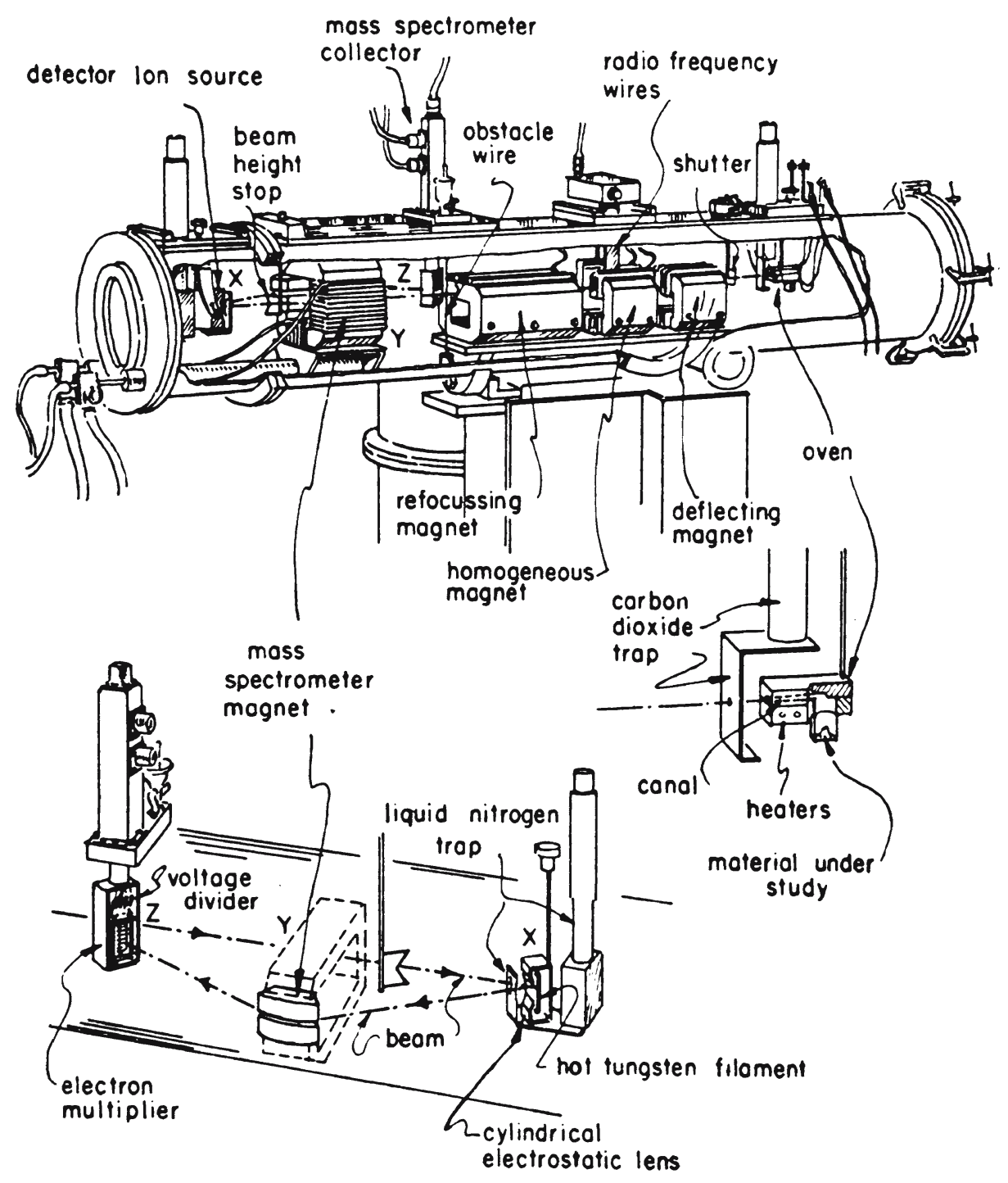

Fig. 10 Atomic beam apparatus used at MIT for radioactive-atom experiments. Note the use of a mass spectrometer in the detecting system [46] (courtesy (C)American Physical Society http:// prola.aps.org/abstract/PR/v76/i8/p1068_1)

Fig. 11, at Princeton University, under Donald Hamilton [48], with which isotopes in the range of minutes could be measured, "off-line," the cyclotron used for the production being just some $50 \mathrm{~m}$ away, Fig. 12.

Obtaining a resonance curve was not trivial because of instabilities in the electronbombardment atomic beam oven. The circular detector buttons were split into 8 sectors. Each sector was exposed for $20 \mathrm{~s}$ at a particular frequency, rotated to the next sector for a background exposure, the frequency changed to the following value, 


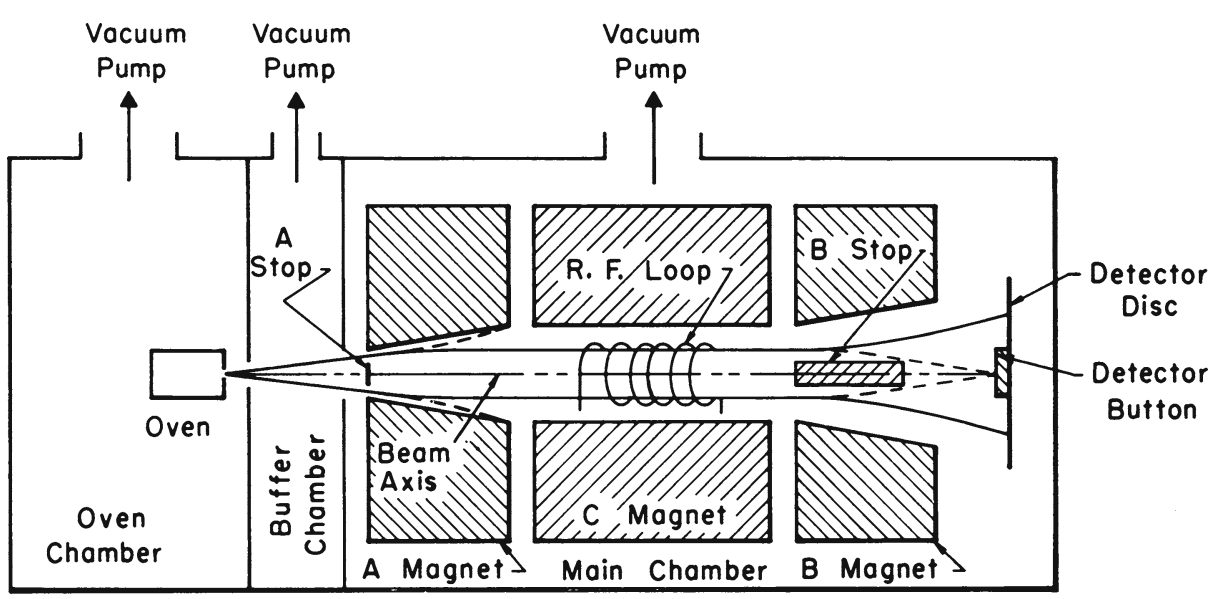

\section{ATOMIC BEAM APPARATUS}

Fig. 11 Princeton focussing ABMR schematic. The A and B magnets have a six-pole geometry. The atoms are collected on detector buttons, which are removed from the apparatus for measuring the collected radioactivity

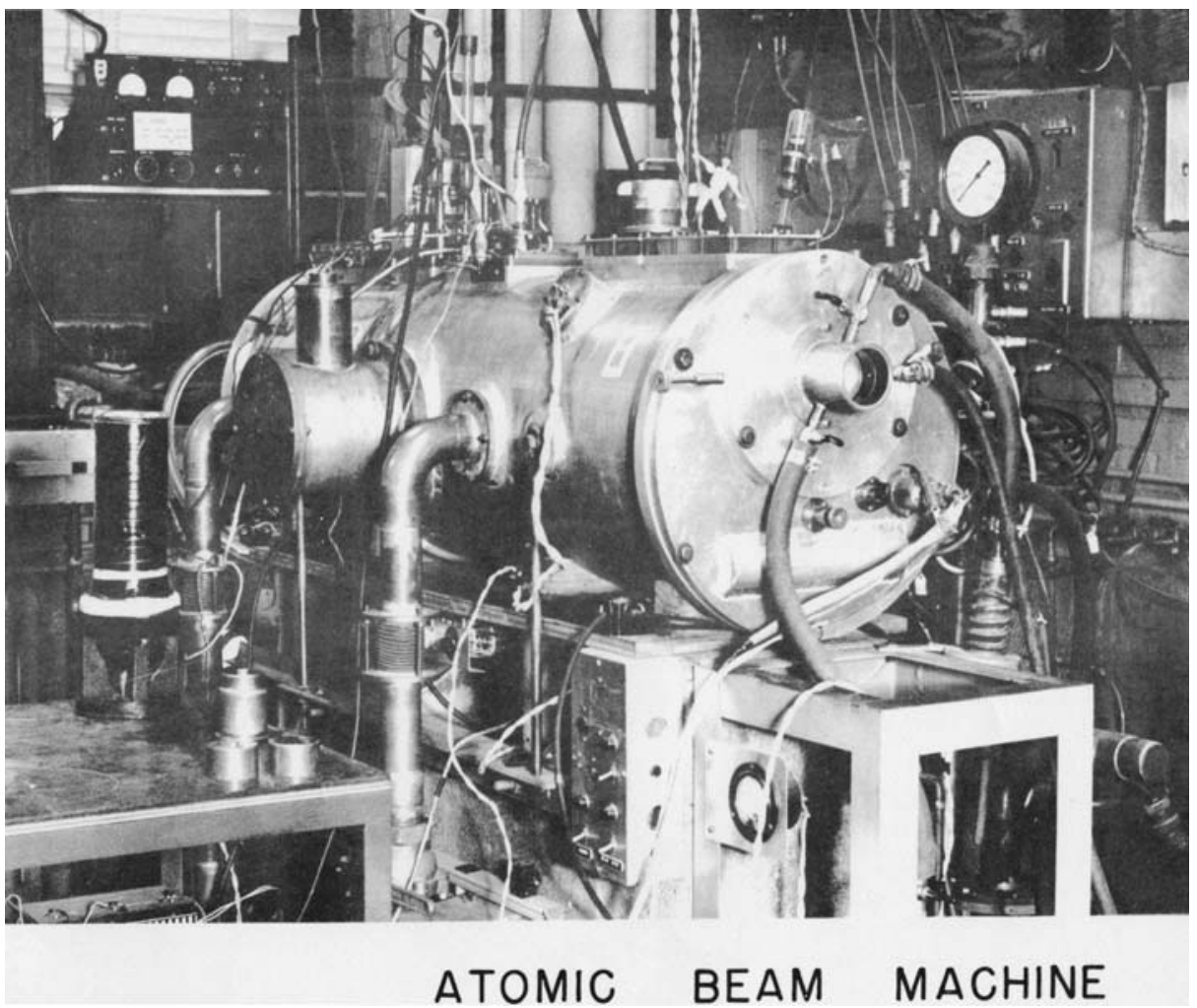

Fig. 12 Photograph of Princeton ABMR. The port through which the detector buttons are inserted into the apparatus is seen above the middle of the end plate 
Fig. 13 Francis Bitter in his MIT laboratory with his technical assistant (with permission, (C) courtesy MIT Museum, [51])
Fig. 14 Jean Brossel (courtesy Laboratoire Kastler-Brossel, Ecole Normale Supérieure)
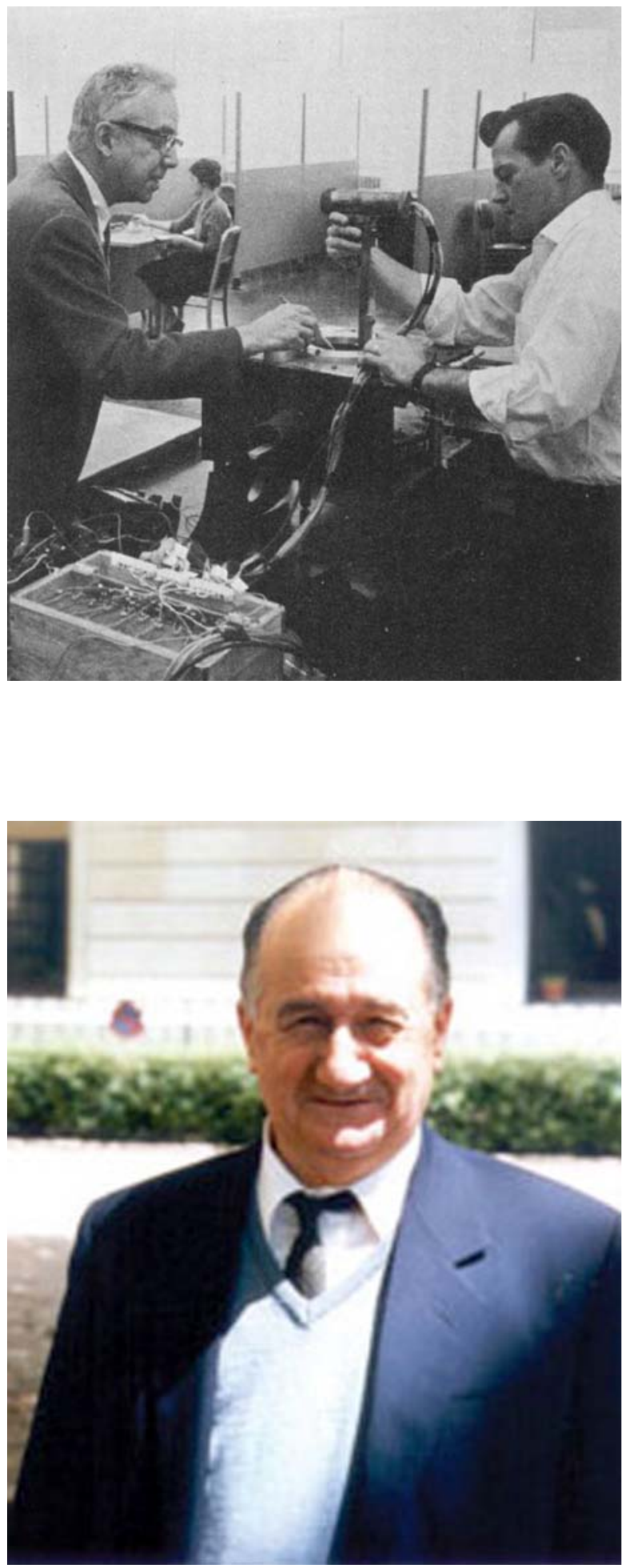
Fig. 15 Double resonance with polarization detection

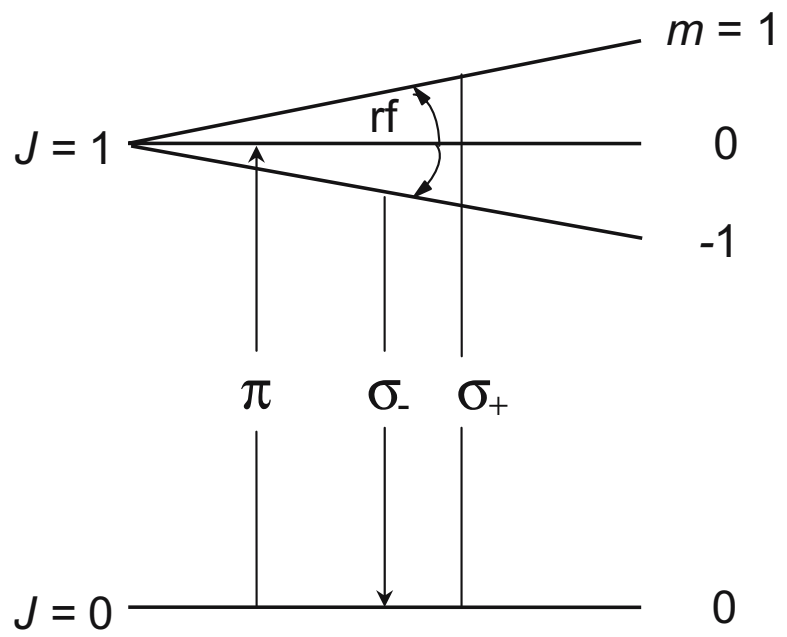

etc. for a total accumulation time of $5 \mathrm{~min}$ on each sector. This was signal averaging at its most primitive!

\subsection{Optical methods}

\subsubsection{Optical double resonance}

After a false start [49] by Francis Bitter, Fig. 13, he and Jean Brossel [50], Fig. 14, developed the "Double-Resonance" method for studying the structure of excited atomic states with great sensitivity [51].

The double resonance consists of an optical resonance followed by an rf resonance, the detection of the latter relying on a change of polarization of the re-emitted light. This is illustrated in Fig. 15: in this example, atoms in a magnetic field in $J=0$ are excited to a $J=1, m=0$ state with use of $\pi$-polarized light. Following rf transitions, $\sigma_{ \pm}$light is emitted.

Much of the work in Bitter's laboratory at MIT for over one and a half decades was devoted to the hfs spectroscopy of stable and radioactive mercury isotopes, ranging from mass number, $A=192$ to 204 . I will touch on the several experimental techniques. An elaboration of the schematic of the polarization double resonance method is shown in Fig. 16 from the work on 23.8-h ${ }^{197 m} \mathrm{Hg}$ of Henry Hirsch [52, 53].

These experiments were done long before the advent, in 1966, of tunable dye lasers [54, 55]. If you look at Fig. 16 carefully, you will note that the tunable light source to produce the first (optical) resonance, either for double resonance or level-crossing experiments, is a ${ }^{198} \mathrm{Hg}$ isotopic lamp placed in a magnetic field. Such "Zeeman" tuning was already used in 1929 by Marcel Schein [56], and was rediscovered by Bitter to become known as the "Bitter magnetic scanning" technique. The energy levels of ${ }^{197 m} \mathrm{Hg}$ in a magnetic field and the transitions used in the double resonance and level-crossing experiments are indicated in Fig. 17. 


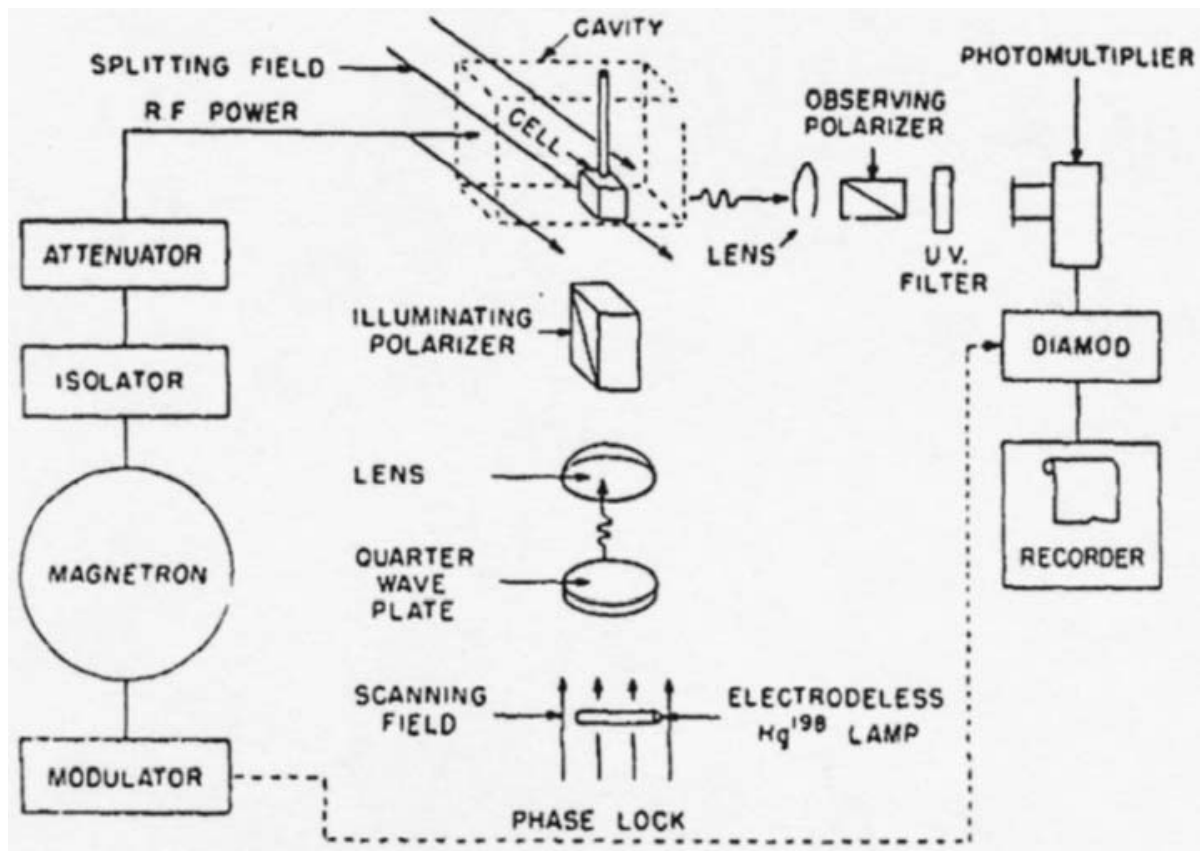

Fig. 16 Double-resonance apparatus schematic (from [52, 53], courtesy of the Optical Society of America)

Fig. 17 Zeeman hfs levels of ${ }^{197 m} \mathrm{Hg}$ (from [52, 53], courtesy of the Optical Society of America)

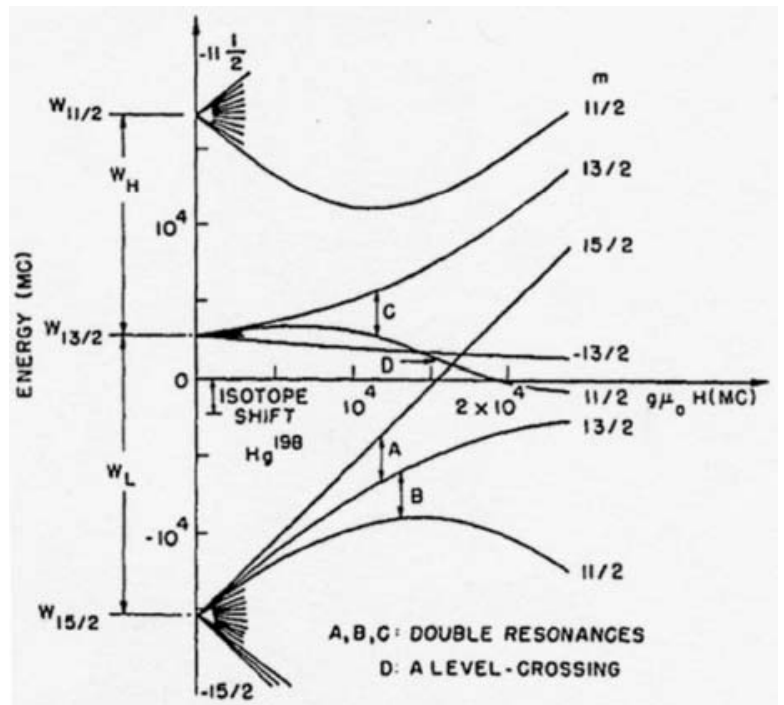

\subsubsection{Double resonance by frequency change}

For special spectra, e.g. in mercury, Robert Kohler $[57,58]$ introduced a new doubleresonance method that depends on a change in frequency in a transition rather than 空 Springer 


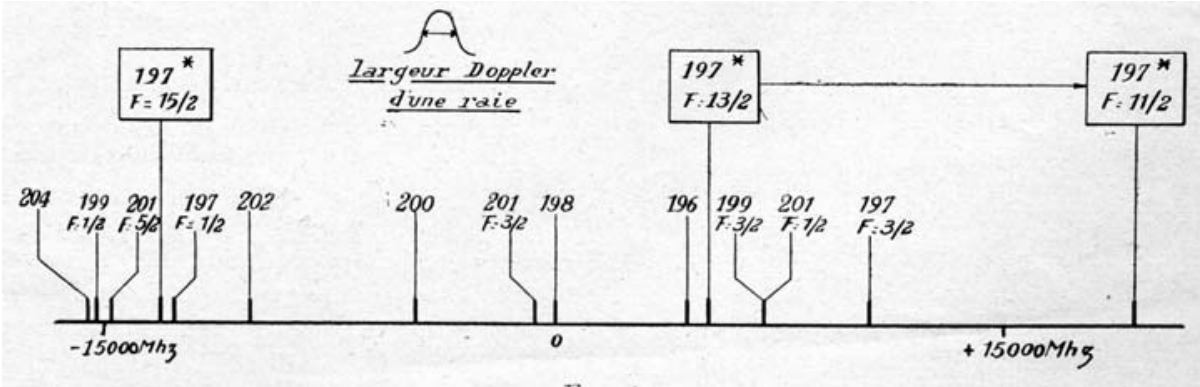

Fig. 18 Hyperfine components in mercury spectrum (from [59], courtesy of the Société Française de Physique)

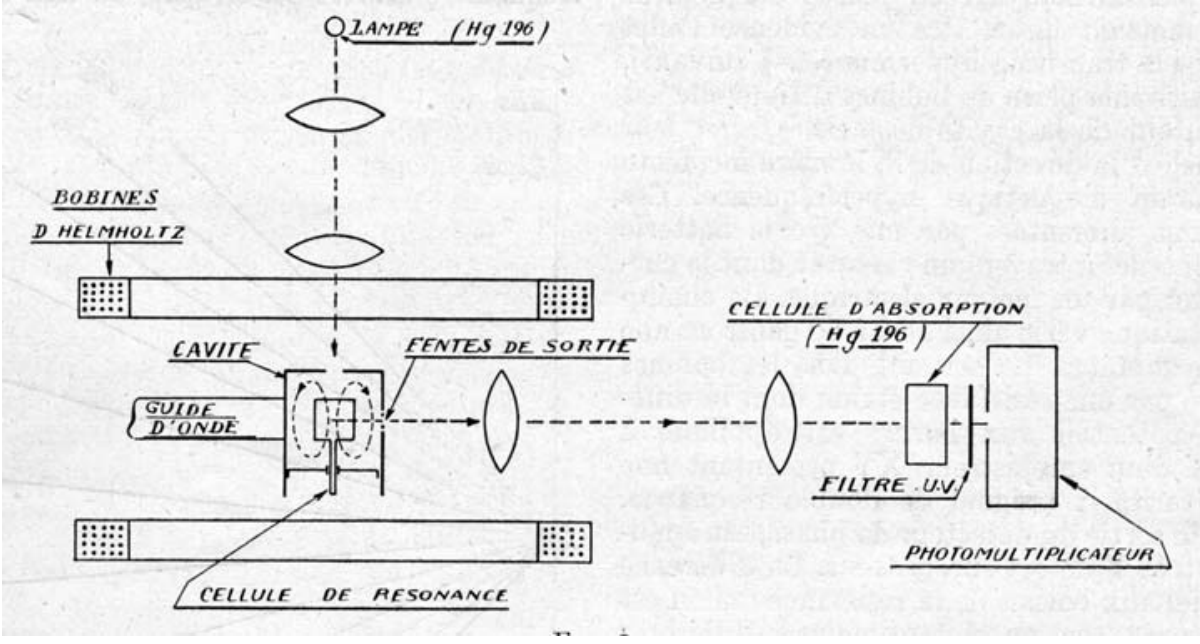

Fig. 19 Experimental setup in the Kohler-type double resonance experiment for ${ }^{197 m} \mathrm{Hg}$ hfs measurement (from [59], courtesy of the Société Française de Physique)

a change in the polarization of the light. The hfs separations have to be much larger than the Doppler widths of the transitions. The scheme was applied by Claude Brot [59], then at MIT, to a precision measuremennt of the hfs separations of ${ }^{197 m} \mathrm{Hg}$, shown in Fig. 18.

The scheme was possible here because of the near coincidence of the $F=13 / 2$ component of ${ }^{197 m} \mathrm{Hg}$ and the 0.15 percent stable isotope ${ }^{196} \mathrm{Hg}$. The latter had to be enriched both for the light source and the absorption cell, Fig. 19.

\subsubsection{Level-crossing spectroscopy}

The scheme for level-crossing spectroscopy is not far removed from Fig. 17, except that changes in the observed intensity of re-emitted light, rather than of the polarization of the light, are observed. We have already cited the theoretical basis, $[28,29]$. Simply, if transition amplitudes from two excited levels to a common lower 


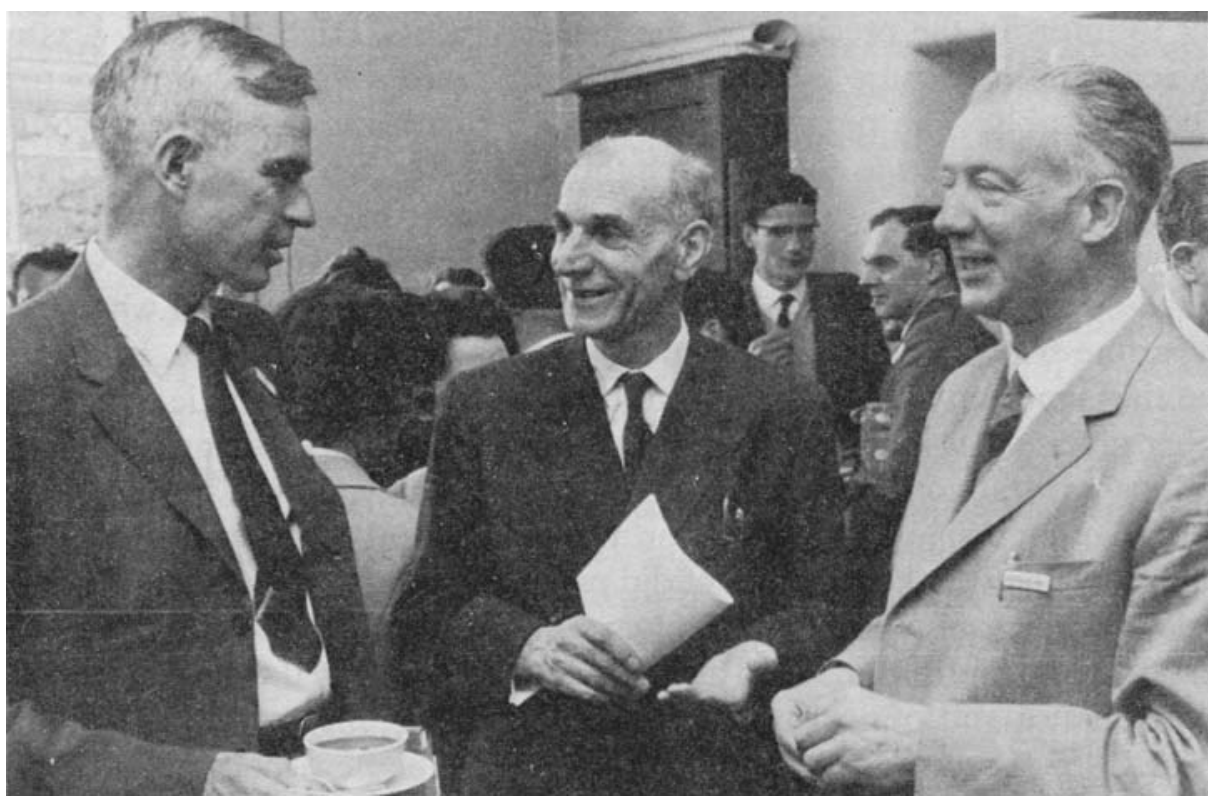

Fig. 20 Willis Lamb, Alfred Kastler, and Hans Kopfermann, with George Series in the background on the right (Photograph by Speck, Heidelberg, courtesy American Institute of Physics, Emilio Segré Visual Archives and International Conference on Optical Pumping, 24-26 April, 1962)

Fig. 21 Optical pumping transitions for mercury isotopes with nuclear spin $I=\frac{1}{2}$, e.g. ${ }^{197} \mathrm{Hg},{ }^{199} \mathrm{Hg}$

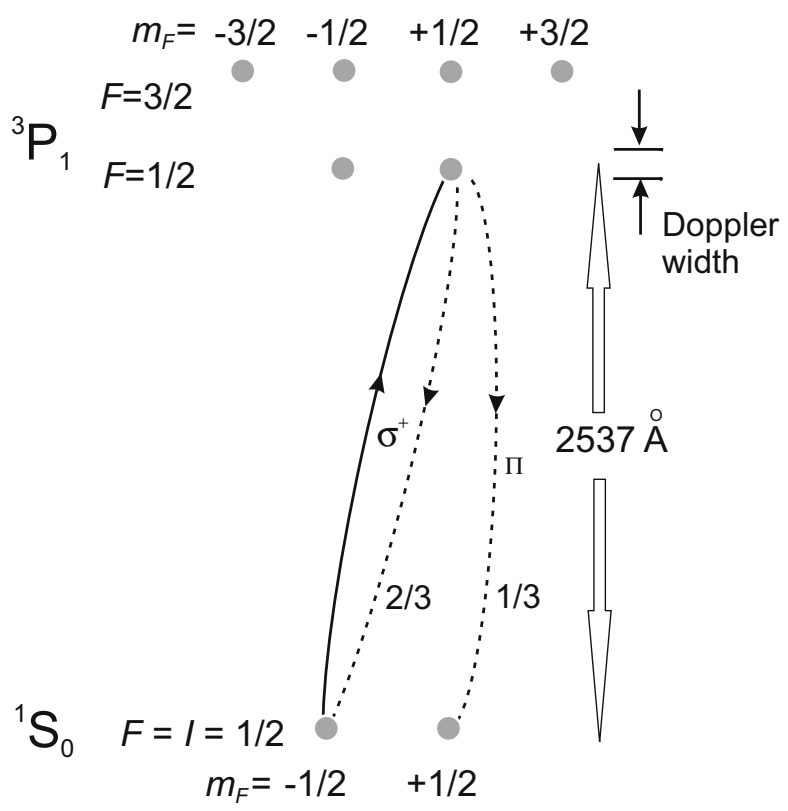




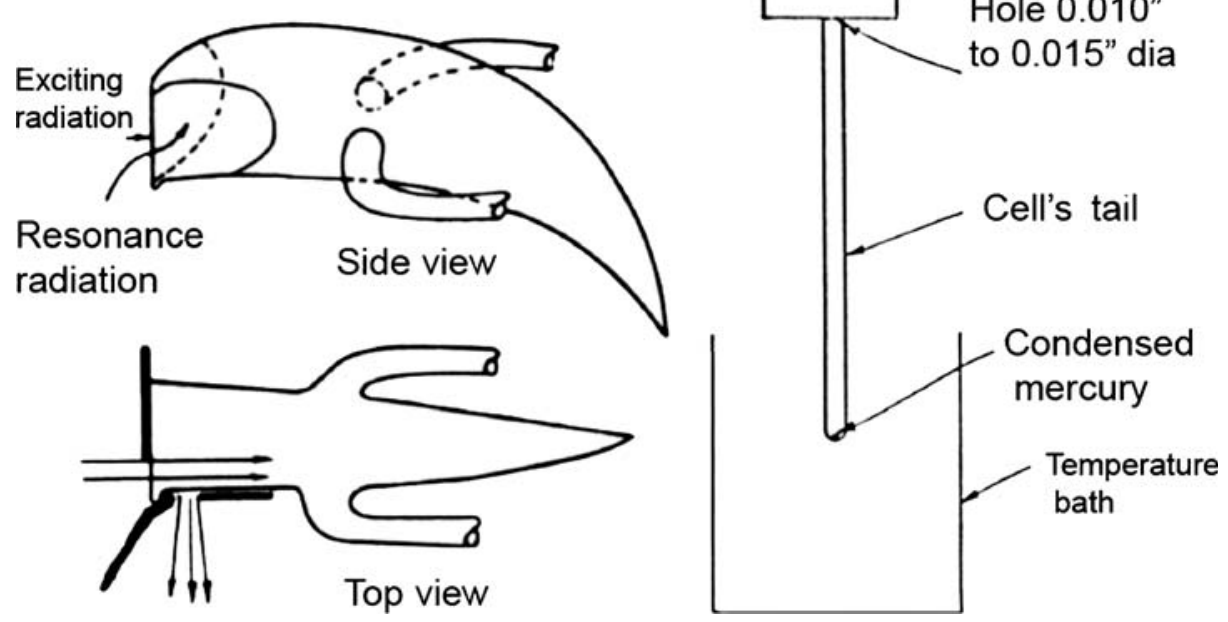

Fig. 22 Left: Wood's horn; right, resonance cell in which optical pumping was successfully observed (see, HHS in [51], p. 289, courtesy MIT Press)

levels are $a$ and $b$, away from the level crossing the intensity is $a^{2}+b^{2}$, while at the level-crossing magnetic field it is $(a+b)^{2}$, leading to an observable interference term. The value of the crossing fields, from which hfs interactions can be obtained, are determined without being limited by Doppler broadening. The method has been applied to several radioactive isotopes, e.g. ${ }^{197 m} \mathrm{Hg}$ and ${ }^{203} \mathrm{Hg}$.

\subsubsection{Optical pumping}

The optical pumping method also dates to this period. It originated with Alfred Kastler, (Fig. 20), in 1950 [60] . We describe below its importance to the measurement of nuclear magnetic moments, in particular radioisotopes.

The basic scheme is shown in Fig. 21.

The mercury is excited from the ground ${ }^{1} \mathrm{~S}_{0}, F=\frac{1}{2}$ state with circularly polarized light to the excited ${ }^{3} \mathrm{P}_{1}, F=\frac{1}{2}$ state. The selection rule $\Delta m=+1$ allows only the $m=$ $-\frac{1}{2}$ state to be excited. The spontaneous re-radiation allows transitions with $\Delta m=$ \pm 1 , thereby populating the $m=+\frac{1}{2}$ sublevel, where the atoms are blocked. Since the ground-state angular momentum is due entirely to the nuclear spin, a corresponding nuclear orientation is produced. Attempts at observing it met with initial failure [61]. The cause was eventually found in the resonance cells that were used. As when work on gas lasers first began, the above optical work also relied heavily on the book of Allan Mitchell and Mark Zemansky (the former at New York University) [62], in particular the resonance cells. The type of cell that was used at first was the "Wood's horn" (named after R.W. Wood), shown in Fig. 22.

In the horn, a large surface of mercury liquid is seen by the oriented atoms and condensation rapidly equalizes the two magnetic substates, $m$. In the cell shown on 


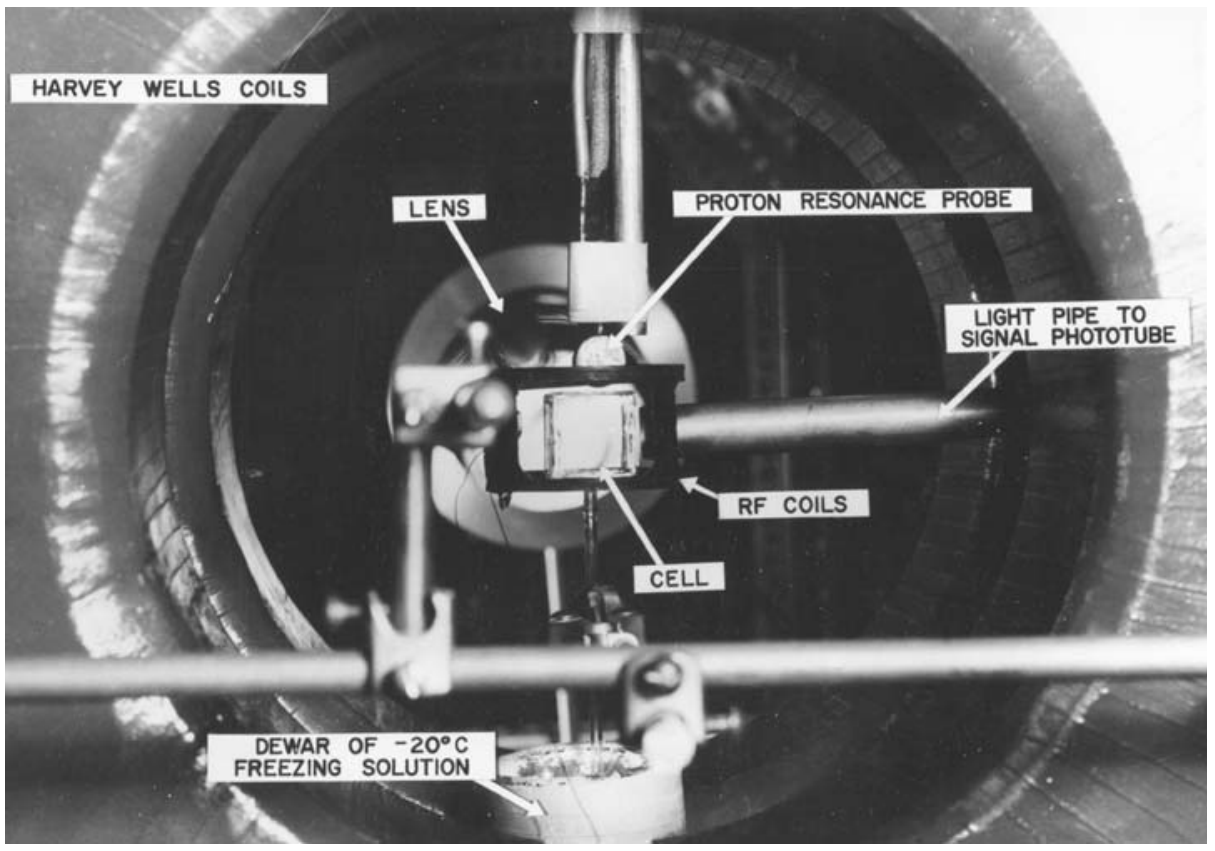

Fig. 23 Optical pumping setup for measurement of ${ }^{195,197} \mathrm{Hg}$ (courtesy W.T. Walter [66])

the right, the atoms do not see the mercury reservoir and relaxation is negligible. An optical pumping experiment with stable mercury isotopes by Bernard Cagnac thus became successful [63-65] and the first measurement of radioisotopes, ${ }^{195} \mathrm{Hg}$ and ${ }^{197} \mathrm{Hg}$ were made [66, 67]. Figure 23 shows William Walter's experimental setup.

At the onset of this section, the importance of the optical pumping experiments to the measurement of nuclear moments with great sensitivity was stated. In Section 2.2.2 the requirement of nuclear magnetic moments, or g-factors, was brought out. For stable isotopes, these can be obtained by nmr in bulk samples, obviously not possible for minute quantities of radioisotopes. Optical pumping, as described in particular for mercury, permits the measurement: while a resonance cell is being pumped it absorbs light. When orientation is complete the vapor becomes transparent. The repopulation of the $m$-levels can be re-established by the nmr transition, and detected by the change in transparency. I should mention that another possibility to obtain the magnetic moment, especially in alkali atoms, is to measure the term in the hfs Hamiltonian that depends directly on the interaction of the magnetic moment with the applied magnetic field. This was used in obtaining the moments in a series of cesium radioisotopes [68].

Optical pumping orientation was successful early on in alkali atoms. Asymmetry in beta decay of oriented ${ }^{21} \mathrm{Na}$ was measured by Otten, Fig. 24, and co-workers [69], a forerunner of a an entire program of measurements on radioactive nuclei at ISOLDE. 
Fig. 24 Ernst Otten: the pioneer in radioactive-atom spectroscopy at ISOLDE (photo by author)

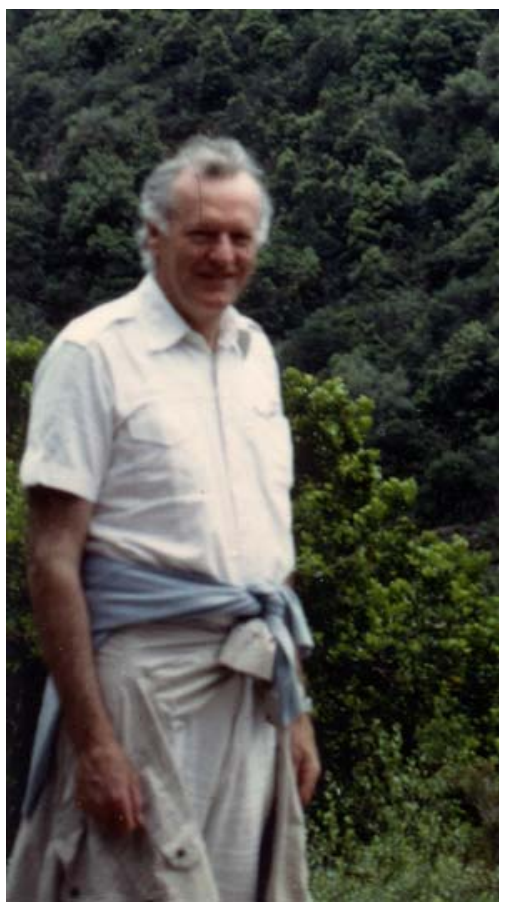

\subsubsection{Radioactive source preparations}

I concentrate on the experiences at MIT and some of our later work: these should serve to give a picture of "off-line" source preparations, and increase our appreciation of doing spectroscopy at ISOL facilities! In most experiments transmutation reactions were used in the production, so as to avoid large stable-isotope backgrounds. For a number of experiments the production was at a cyclotron with use of a ( $p, x n)$ nuclear reaction. For the mercury isotopes, this was straightforward with the use of a gold target. After irradiation, a cell or electrodeless discharge lamp was produced with a system as shown in Fig. 25.

In a few cases a nuclear reactor was used to produce the radioisotope, but this demanded subsequent mass separation. For other elements, radiochemistry was required, and for thallium the development of a liquid mercury target acceptable to cyclotron establishments! Typical lamps and cells are shown in Fig. 26. In use, the lamps frequently necessitated to be heated with the use of a propane torch to drive the few atoms back from the quartz surface into the electric discharge.

\subsubsection{Grating optical spectroscopy}

Most of the optical spectroscopy of the radioactive isotopes was done with the use of a 10-m focal length (slightly shorter at NYU) Czerny-Turner two-mirror monochromator, Fig. 27.

A $25-\mathrm{cm}$ wide ruled diffraction grating, 300 lines $/ \mathrm{mm}$, blazed at $60^{\circ}$, was the heart of the instrument. A photograph is shown in Fig. 28. 


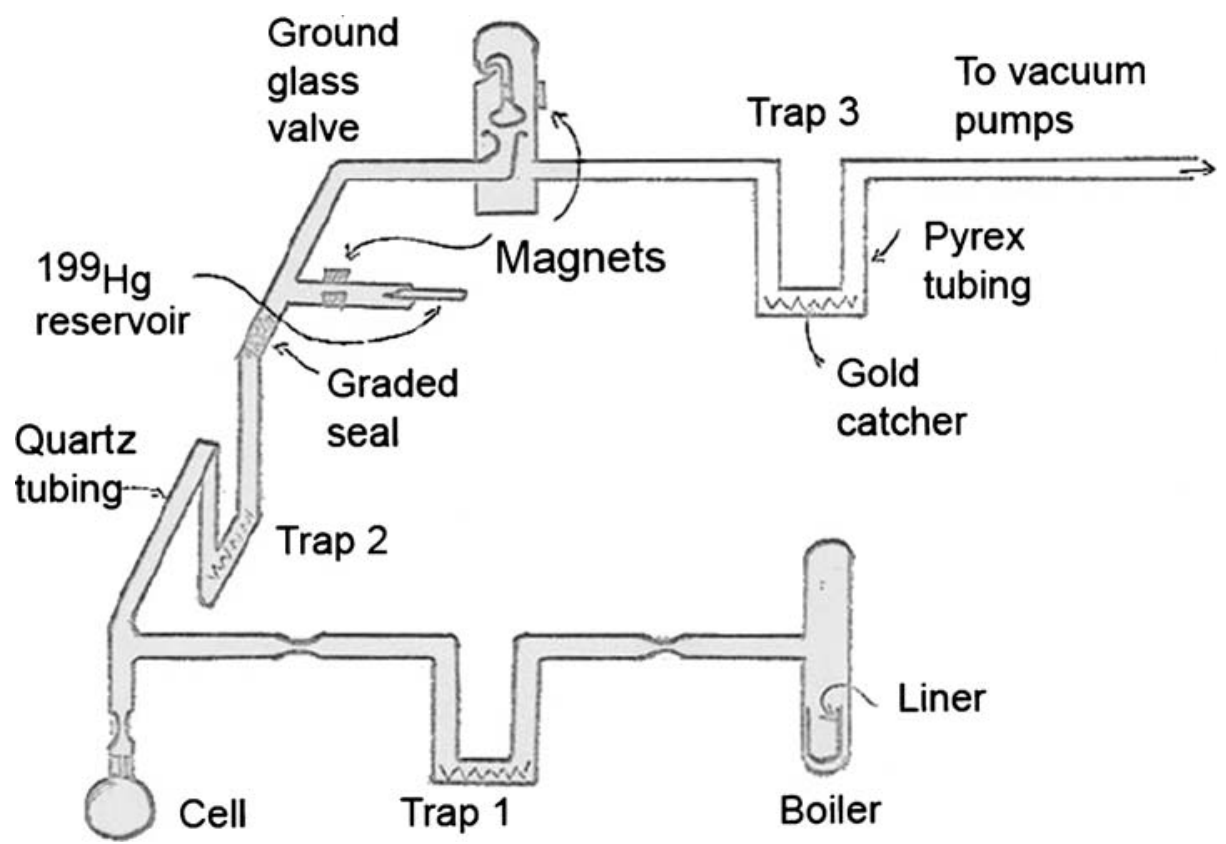

Fig. 25 The mercury in the irradiated gold foil is melted with an induction heater, driven to gold catchers, and finally to the resonance cell or lamp

Fig. 26 Optical-pumping cell and spherical electrodeless lamps. Scale is in $\mathrm{cm}$

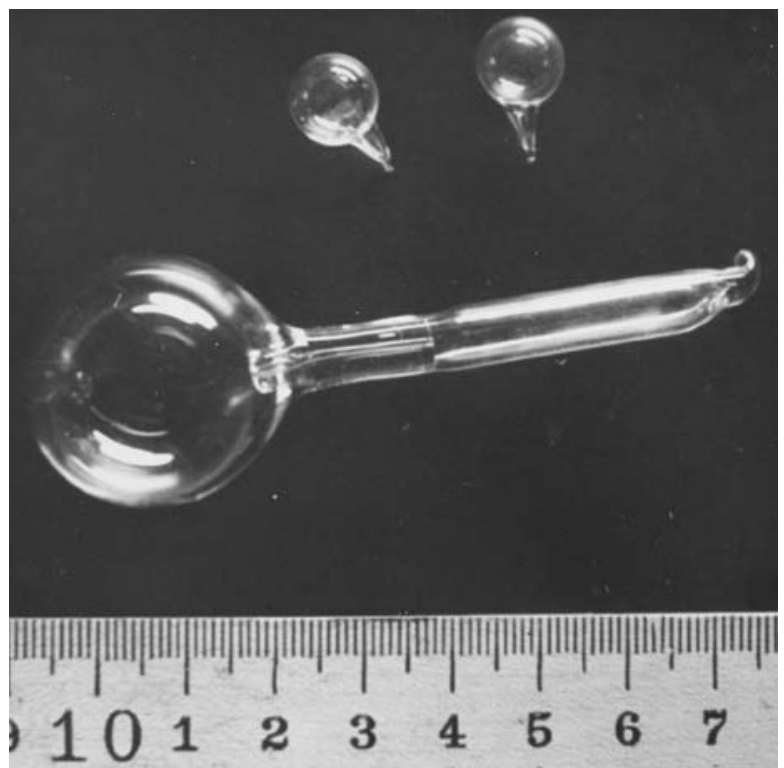

Photographic or photoelectric detection was used, as well as an image intensifier. Since the preparation time for the fabrication of a spectral lamp took about two weeks, and the lamps had a limited lifetime (not just radioactively, but frequently 基 Springer 
Fig. 27 Grating spectrometer, schematic. The grating spacing is denoted by $d$

Fig. 28 1-slit, 2-diffraction grating, 3-photographic plate holder

\section{Spectrograph}

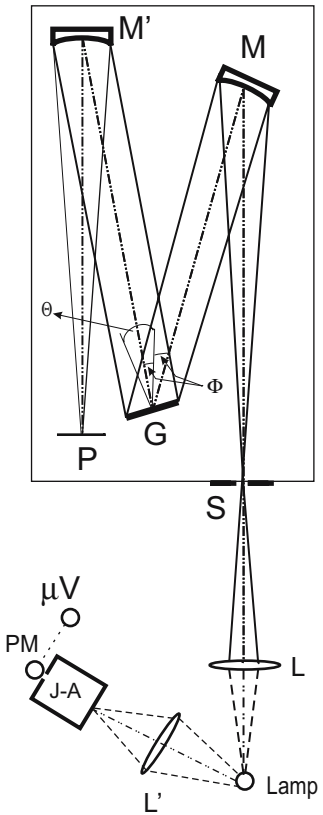

L - Quartz lens, $f=25 \mathrm{~cm}, d=3.8 \mathrm{~cm}$

S - Slit, $30-40 \mu \mathrm{m}$

M - Collimating mirror, $10 \mathrm{~m}$ focal length, $25 \mathrm{~cm}$ diameter

$\mathrm{G}$ - Diffraction grating, $25 \mathrm{~cm}$ wide, $\mathrm{d}=3.33 \mu \mathrm{m}, \theta_{\text {blaze }}=61^{\circ}$

M' - Camera mirror, $10 \mathrm{~m}$ focal length, $25 \mathrm{~cm}$ diameter

P - Plate holder, uses $25 \mathrm{~cm}$ spectroscopic plates

L' - Quartz lens

J-A - Jarrel-Ash monochromator for monitoring lamp

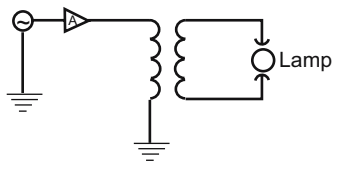

Lamp operation

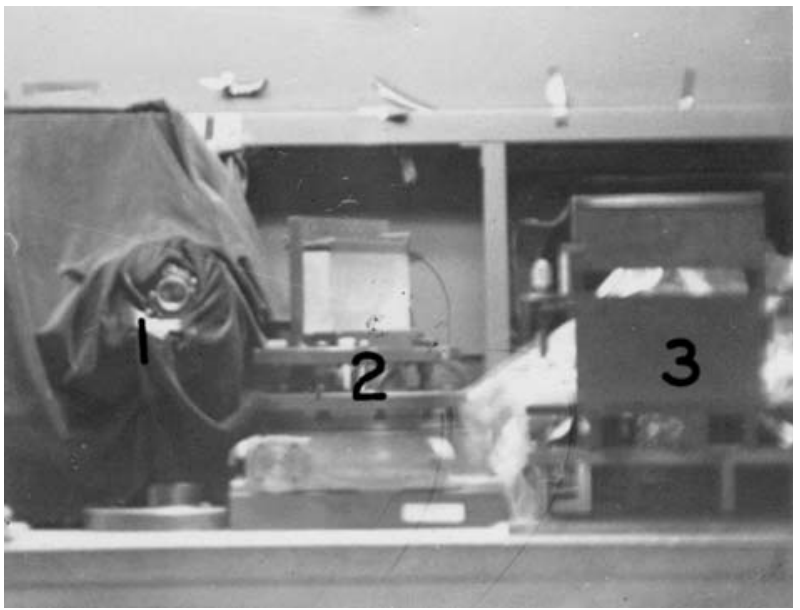

they may have lasted only from a few minutes to hours because of the atom "cleanup" in the discharge), additional camera mirrors and detectors were frequently set up to record simultaneously the spectra of several wavelengths of interest. Absorption spectroscopy was advantageous in atom conservation. A spectrum is shown in Fig. 29.

As a transition to laser spectroscopy, one can bring out the instrumental broadening of grating spectroscopy by comparing its spectrum of $32.9-\mathrm{y}{ }^{207} \mathrm{Bi}$ to one obtained with use of laser scanning, Fig. 30. 
Fig. 29 Photographic absorption spectrum of ${ }^{205} \mathrm{~Pb}$ and of natural lead

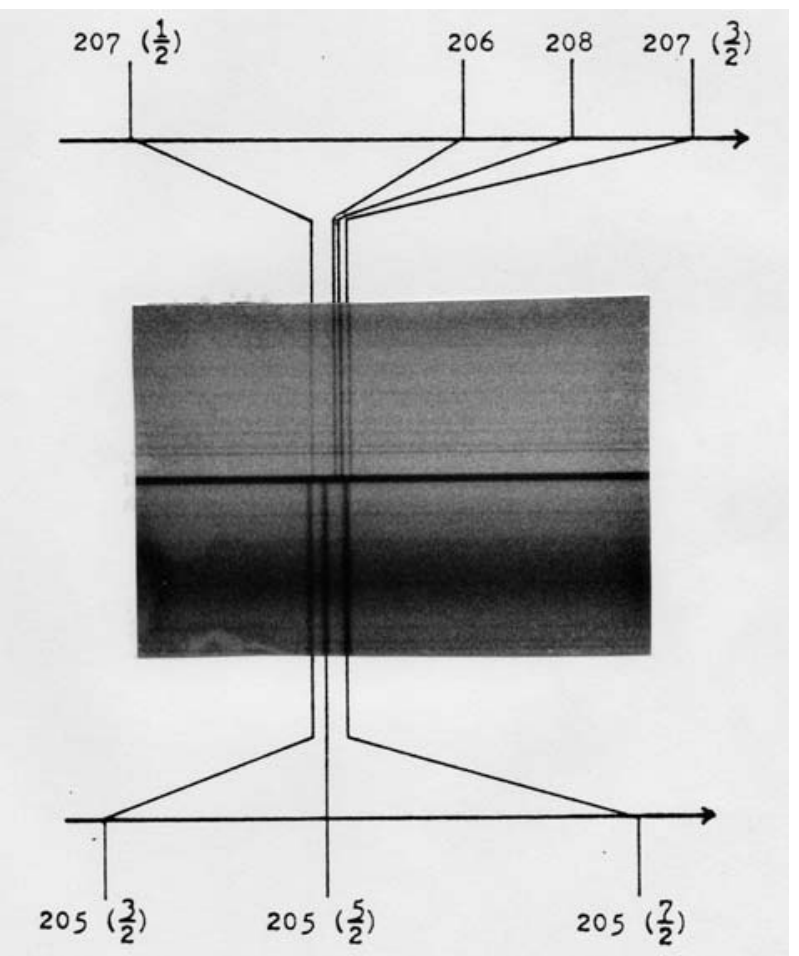

\subsection{On-line experiments}

I will restrict this overview to experiments at ISOLDE (CERN) as they are representative of developments elsewhere in the world. Work in laser-type spectroscopy is active in many laboratories: TRIUMF (Canada), GANIL and ORSAY (France), GSI, Mainz (Germany), Manchester (UK), ORNL, ANL, Stony Brook (USA), JINR (Dubna), Gatchina, Troitzk (Russia), Jyväskylä (Finland), Louvain-la-Neuve, Leuven (Belgium). New facilities are on the way at Lanzhou (China), INS (Japan). Others, that made early contributions to radioactive-atom spectroscopy, are no longer active in this field (Berkeley, Brookhaven, Harwell, Karlsruhe, Sussex). A major facility, EURISOL, is presently under study. There are comprehensive reviews, e.g. Otten, Kluge, Billowes and others [2,4-8]. "On-line" refers to experiments done on isotopes directly as they are produced at the accelerator - though the isotopes may sometimes be first collected, e.g. in resonance cells.

\subsubsection{RADOP}

Nuclear orientation by optical pumping and detection of asymmetry in beta decay were already discussed in Section 3.2.4. This work was intensified at ISOLDE by Jochen Bonn, Gerhard Huber, Jürgen Kluge, and Ernst Otten in a series of experiments named RADOP (radioactive detection of optical pumping) [70], which allowed the detection of sudden deformations in neutron-deficient isotopes of 
Fig. 30 A position of hfs components of ${ }^{207} \mathrm{Bi}$ and ${ }^{209} \mathrm{Bi}$. B Dispersive spectrum. C Laser excitation-fluorescence spectrum. D Frequency markers (Zuyun Fang, PhD Thesis, NYU, 1988)
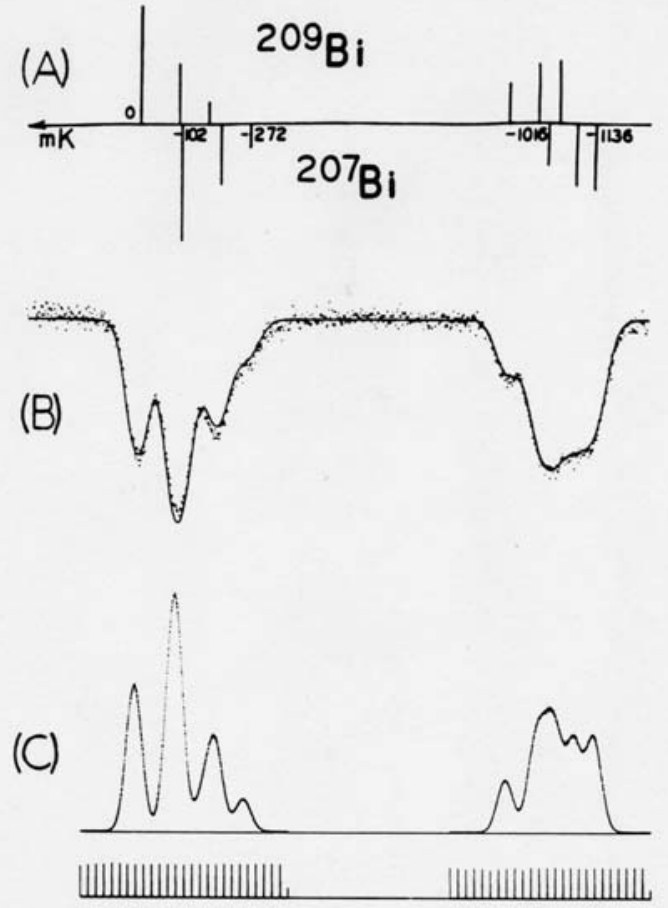

(D)

mercury [71]. In these early experiments, a Zeeman-tuned spectral lamp was still used for the optical pumping, Fig. 31.

\subsubsection{Atomic beams}

An extensive series of ABMR, non-laser experiments on cesium isotopes was done by Curt Ekström to obtain hfs systematics [72]. Use of the tuned laser as the spectrometric instrument appeared in atomic beam experiments by the Orsay group [73] on a long series of sodium isotopes. They could take pride in the measurement by this method of the first optical spectrum of the element francium [74]. Their experimental setup [75] is shown in Fig. 32.

The last of the atomic beam experiments, Fig. 33, is again of the magnetic resonance type, but in which the laser is used for state selection. It was designed for the measurement of the Bohr-Weisskopf effect in a long series of cesium isotopes [76], in an analogous way to those in [68]. Fig. 34 is a photo of H.T. Duong, a prime designer of this experiment.

As with other on-line atomic beam experiments, the ions from ISOLDE have to be neutralized and thermalized. While this was done successfully in prior experiments by stopping, neutralizing the ISOLDE ion beam on an yttrium surface, and reevaporating to produce the thermal atomic beam, in this case the surface effect 


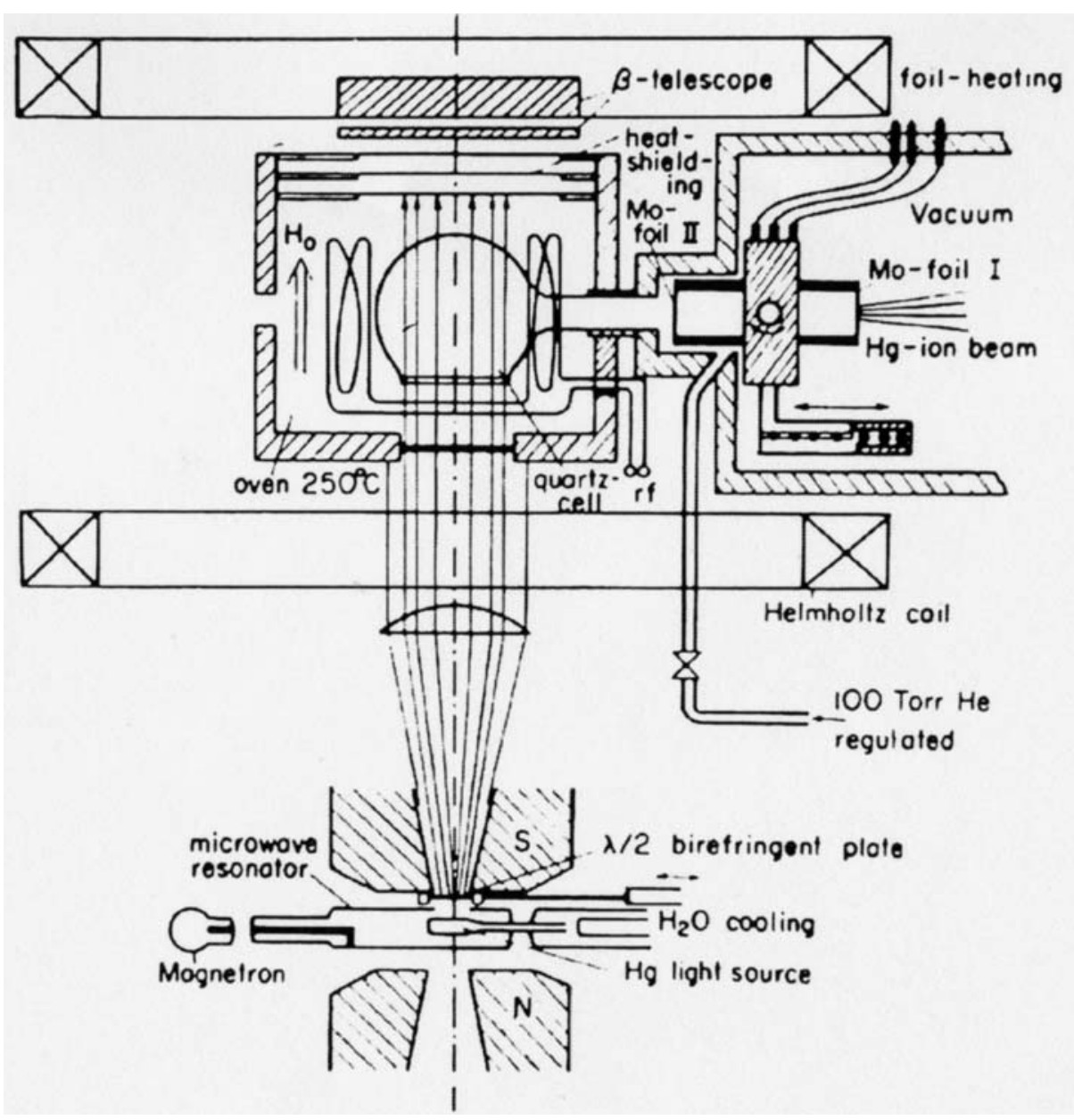

Fig. 31 RADOP setup for measurement of $2.4-\mathrm{m}^{187} \mathrm{Hg}$ (from Optical Pumping of Neutron Deficient ${ }^{187} \mathrm{Hg}$ [70], (C1971, with permission of Elsevier)

was not reliable. After considerable efforts the neutralizer, shown in Fig. 35, finally allowed the precision measurement of the hfs of 1.64-m ${ }^{126} \mathrm{Cs}$. This Bohr-Weisskopf study awaits the future.

\subsubsection{Collinear, RIS}

Collinear laser spectroscopy and resonance ionization (mass) spectroscopy (RI(M)S [77]) laid the groundwork for many experiments, a number of which are reported at the VII International Workshop Laser 2006. Collinear laser spectroscopy originated in Mainz, where the initial experiments were performed [78]. A schematic of the experiment as set up at ISOLDE [79] is shown in Fig. 36.

In the acceleration process, the velocity spread of the atom source is reduced to give Doppler-free spectroscopy. With a fixed-frequency laser, the hyperfine spectrum 基 Springer 


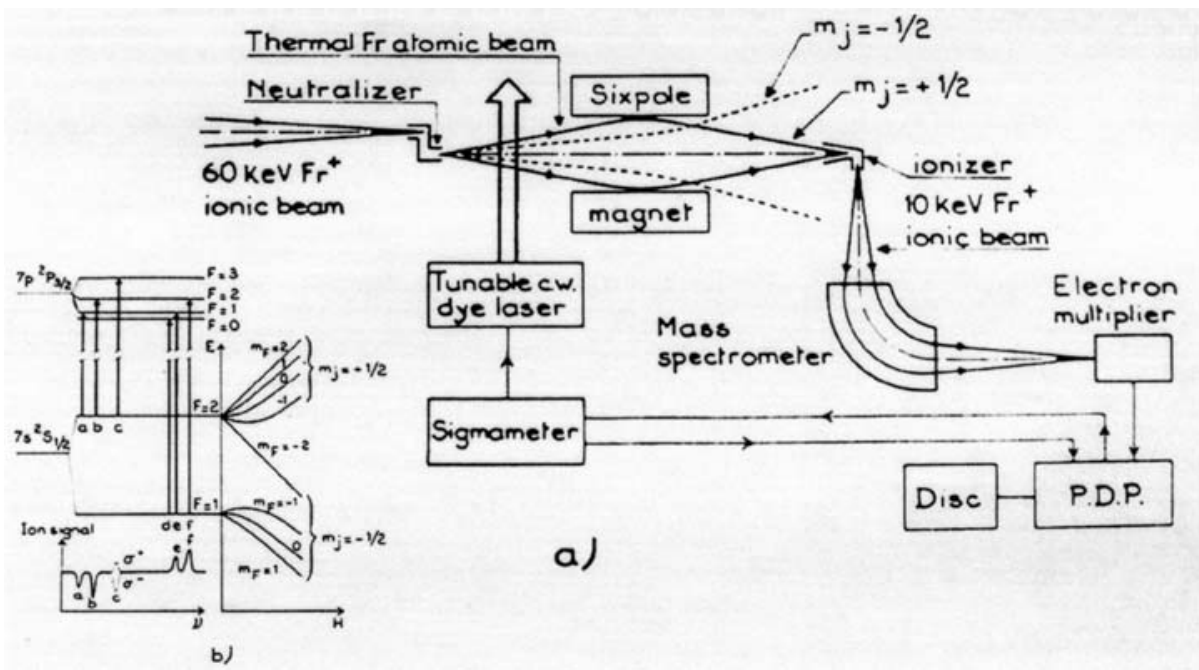

Fig. 32 a) Schematic of apparatus for the D2 francium-line measurement. b) Signals, exhibiting optical pumping effects in transitions (adapted from [75]; courtesy @American Physical Society, http://prola.aps.org/abstract/PRC/v23/i6/p2720_1)

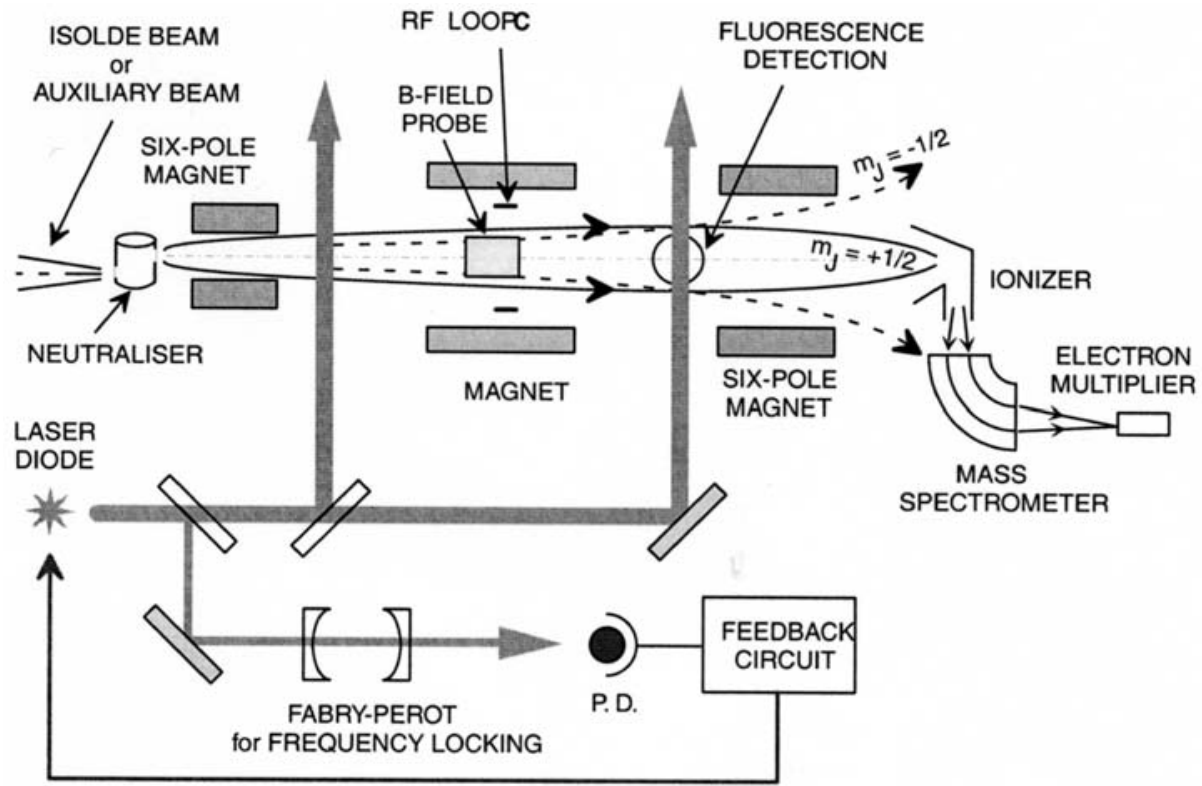

Fig. 33 ABMR apparatus with laser state selection (from Precision $h f_{s}$ of ${ }^{126} C s\left(T_{1 / 2}=1.63 m\right.$ ) by $A B M R$ [76]; (C2005, with permission of Elsevier)

can be tuned across that frequency, and, in the figure shown, the fluorescence detected. An offshoot is the COLLAPS collaboration experiment in which the collinear setup ends with a crystal in which the selected atomic beam is embedded. Beta-decay asymmetry is then used to detect the resonance frequencies. Magda 
Fig. 34 H.T. Duong (courtesy Jacque Pinard)
Fig. 35 Orthotropic source: ions which are not neutralized can be returned to the yttrium surface for more chances of neutralization (following design of T. Dinneen, A. Ghiorso, H. Gould, Rev. Sci. Instrum. 67(1996)752; from Ref.[76], (c) with permission of Elsevier, as in Fig. 33)
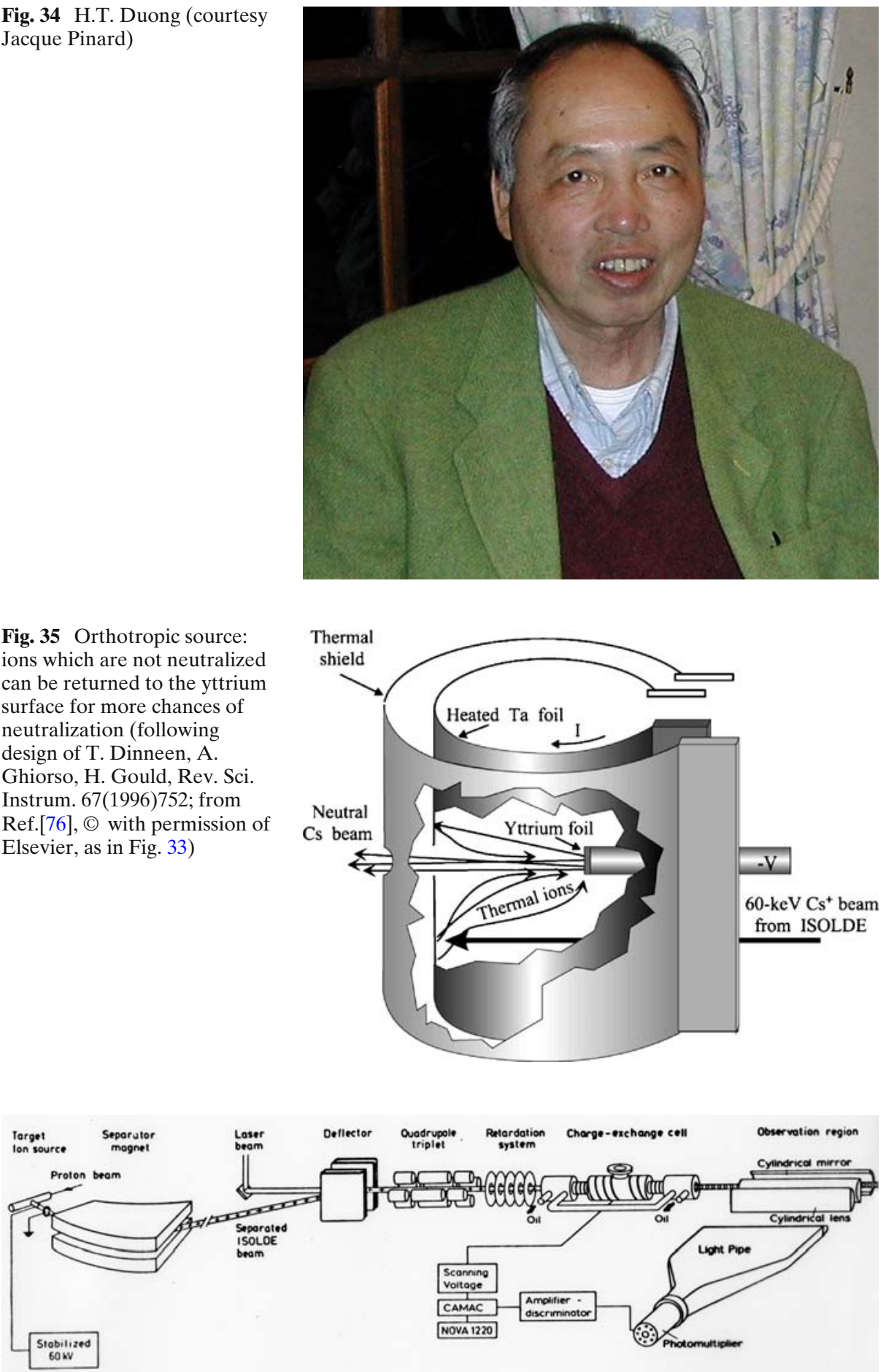

Fig. 36 Collinear laser spectroscopy schematic (adapted from Ref. [79], courtesy of Elsevier) 悬 Springer 


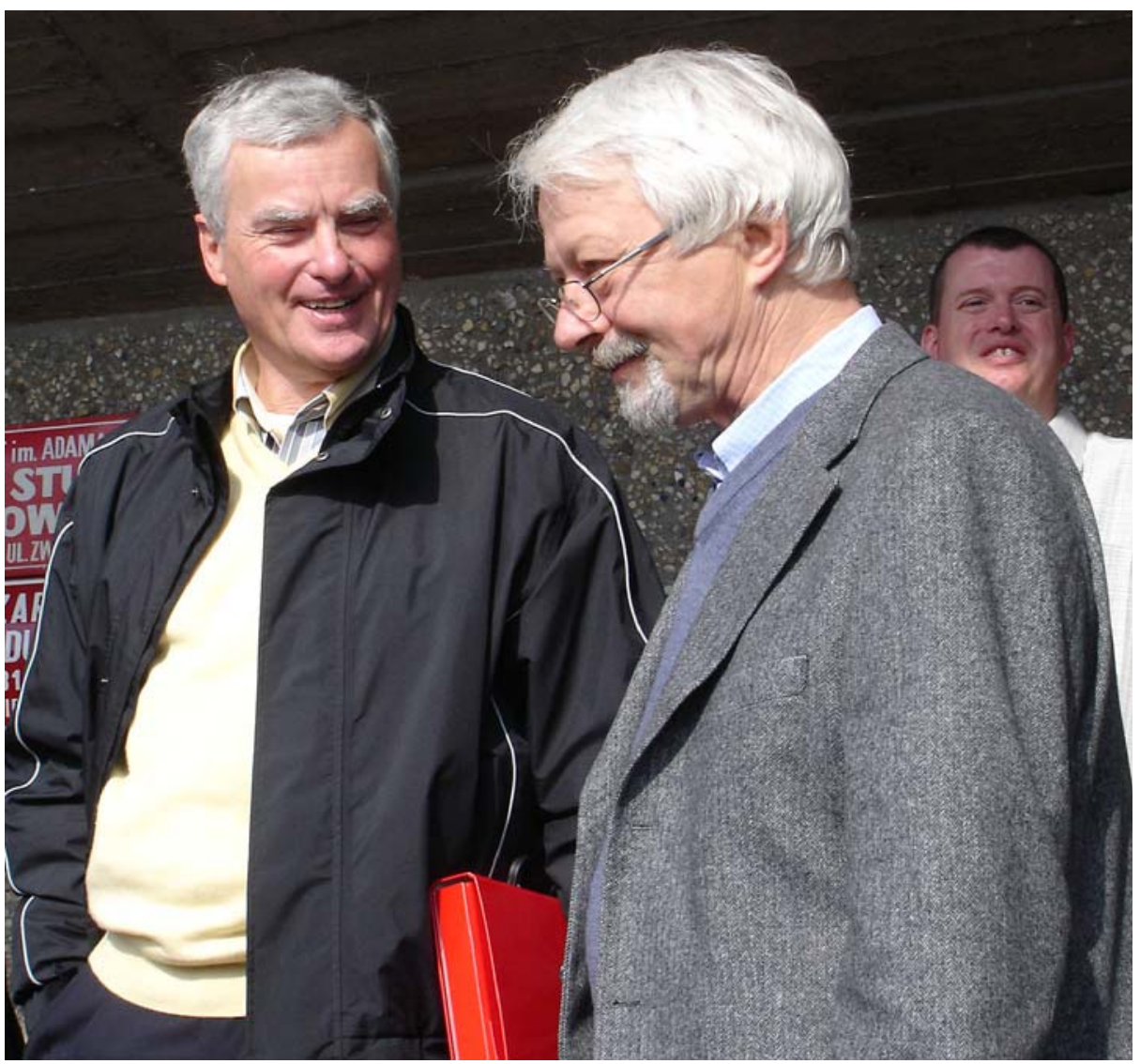

Fig. 37 Rainer Neugart, a pillar of ISOLDE laser spectroscopy! Standing on the left, Ken Ledingham, and in the background, Paul Campbell (courtesy H.-J. Kluge)

Kowalska and Kieran Flanagan are presenting results of experiments with these techniques in this Workshop. I add one more collinear laser spectroscopy experiment: the measurement of the francium D1 line [80]. This was another example of a difficult laser experiment: searching for the wavelength of an optical transition over a wide wavelength region with a fine-tooth comb. In this respect, the old-fashioned diffraction grating spectroscopy has the advantage of displaying lines in large spectral regions! At this point it is good to give particular mention of Rainer Neugart, Fig. 37, who has been intimately associated with the success of a good many of these experiments.

RI(M)S, which was initiated independently at ISOLDE, Orsay, and Gatchina, developed later at CERN into the COMPLIS (COllaboration for spectroscopy Measurement using a Pulsed Laser Ion Source) experiments for the study of refractory elements not produced directly as an ISOL beam. The atoms studied are radioactive daughters of beam ions implanted in graphite, desorbed by a high-power laser pulse, followed by resonance ionization spectroscopy, and time-of-flight detection, Fig. 38. These were substantially efforts by Mainz, McGill, Orsay, Gatchina, Troitzk, and 


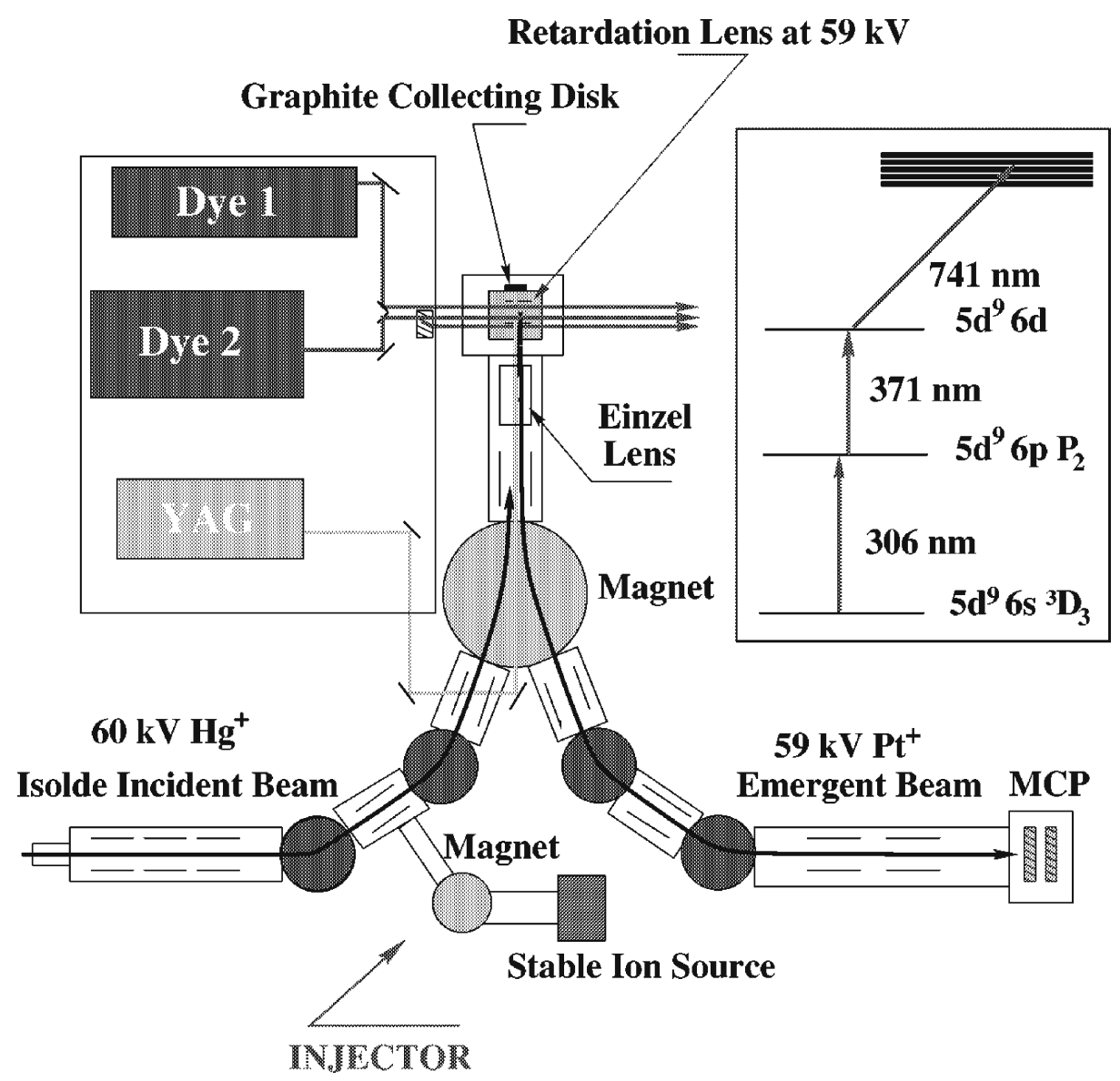

Fig. 38 COMPLIS experiment (from Ref. [86]). The transitions shown are for platinum

ISOLDE [81-86]. Rosa Sifi, Fig. 39, presents current work on tellurium isotopes at this Workshop.

\section{Selected results}

Atomic spectroscopy contributed many early data on nuclear spins, nuclear size, magnetic dipole and electric quadrupole moments which were important in the development of nuclear models and interactions. Although we are treating here mainly radioactive atoms we want at least to mention spectroscopic experiments that had fundamental implications: the quadrupole moment of the deuteron in revealing the tensor force in nuclei, the Lamb shift and the value of the gyromagnetic ratio of the electron in showing effects of quantum electrodynamics. But many of the spectroscopic experiments that we discuss led more to systematic properties from which one hopes to extract detailed nuclear data. 


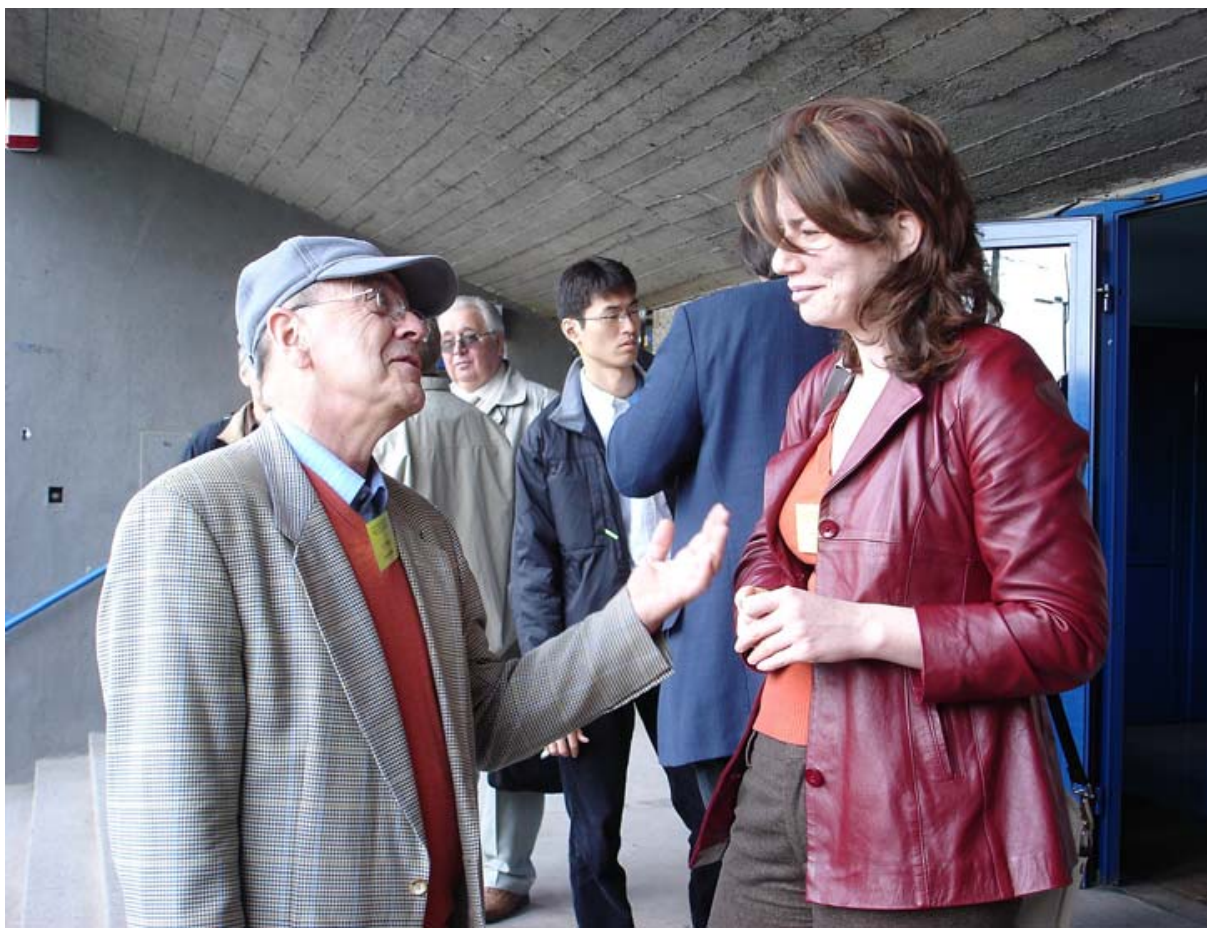

Fig. 39 From the most senior in the field of radioactive spectroscopy, Gerhard Huber, to the youngest, $\mathrm{PhD}$ candidate Rosa Sifi (courtesy H.-J. Kluge)

\subsection{Isotope shifts and nuclear charge distributions}

It is work with radioactive isotopes that has permitted systematic studies over large ranges of isotopes. A spectacular result was the shape staggering in the nuclear radii of mercury isotopes [87], shown in Fig. 40.

Another systematic study is of isotope shifts crossing magic numbers. A typical example, for cesium [88], ${ }^{3}$ is shown in Fig. 41.

As we point out in Section 4.2, an effect of the magic-neutron isotope, ${ }^{137} \mathrm{Cs}$, also appears in the Bohr-Weisskopf effect.

\subsubsection{Odd-even staggering}

It has been observed since the early days of isotope shift studies that the addition of a single neutron to an even-number neutron isotope generally produces less than one

\footnotetext{
${ }^{3}$ See, also, Otten [4].
} 
Fig. 40 Isotope shifts in mercury isotopes reflecting changes in the mean square radii (from Ref. [87]). The sudden changes for the lightest isotopes have been found to correspond to changes in deformation (courtesy (C)American Physical Society, http//prola.aps.org/abstract/ PRL/v39/i4/p180_1)

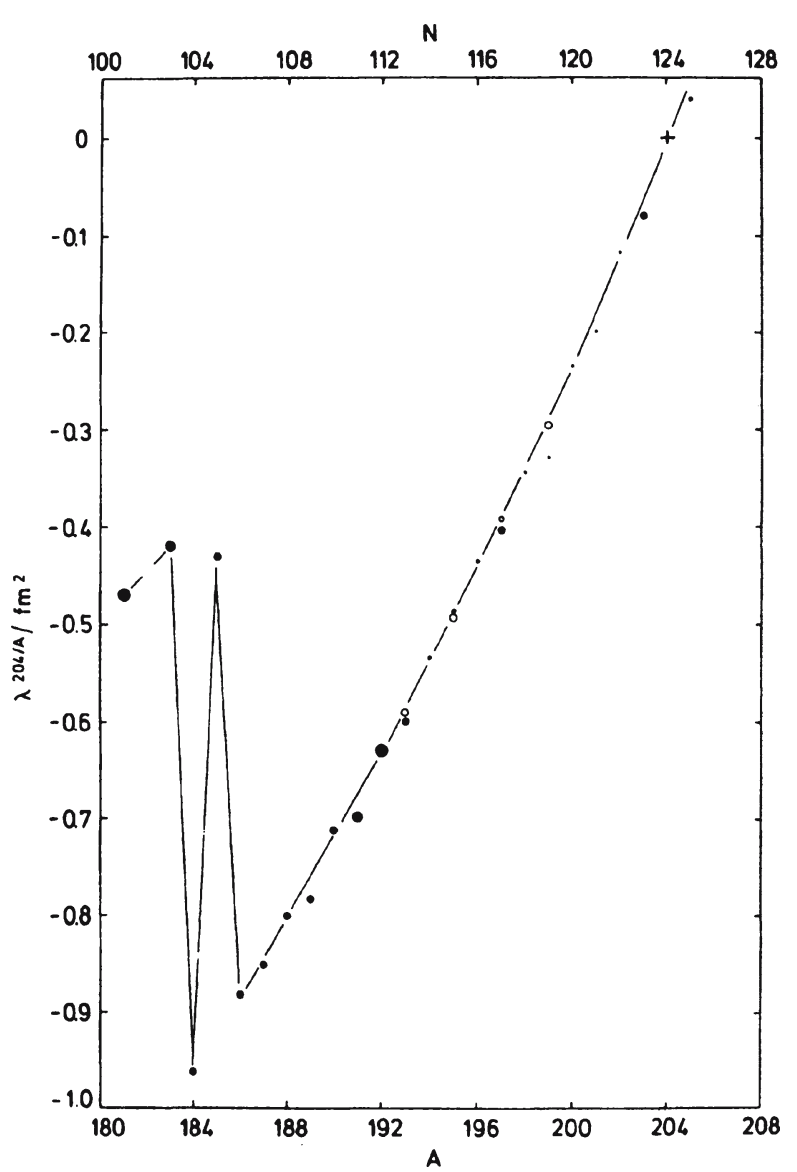

half of the shift caused by the addition of a neutron pair. We have described this by a "staggering parameter",

$$
\gamma \equiv \frac{<r^{2}>_{N+1}-<r^{2}>_{N}}{\frac{1}{2}\left[<r^{2}>_{N+2}-<r^{2}>_{N}\right]} .
$$

$N$ refers to an even-neutron number nucleus. The parameter $\gamma$ is more sensitive to details in the variations of the isotope shifts than what we can see in a plot, such as Fig. 41. In fact, with Jürgen Kluge and Dieter Proetel [89], we found possible to account for the trend of the odd-even staggering parameters, $\gamma$, for the mercury nuclear isomers. Fig. 42 is a photo of Kluge. He has been a prime mover in many important developments of modern radioactive-atom spectroscopy, and now in experiments on precision mass measurements with traps.

We have approached isotope shifts from the point of view of relative shifts, which allows us to compare isotonic shifts, as shown in Fig. 43, from [90]

This picture has been extended recently to radium and radon, with continuing isotonic similarities. The possibility of obtaining insight into the nuclear-neutron interaction from these measurements is being studied with Kieran Flanagan. 
Fig. 41 Cesium isotope shifts: note break in $\Delta<r^{2}>$ at the magic number $N=82$ (from [88])

Fig. 42 Jürgen Kluge (photo by author)
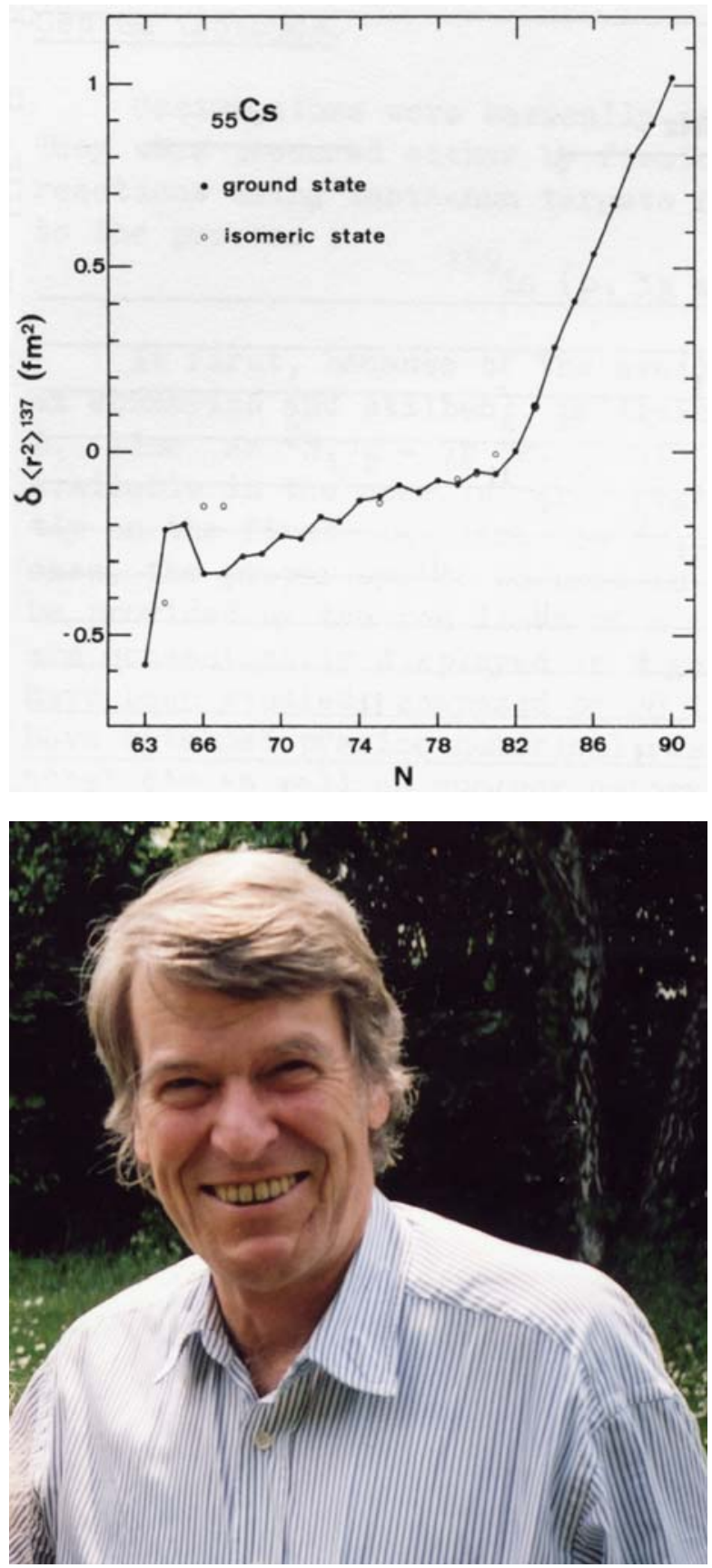

There are other systems which I have to pass over, such as isomer shifts in fission isomers and shifts in lithium halo nuclei. The physics that one can expect to obtain from isotope shifts is not exhausted! 

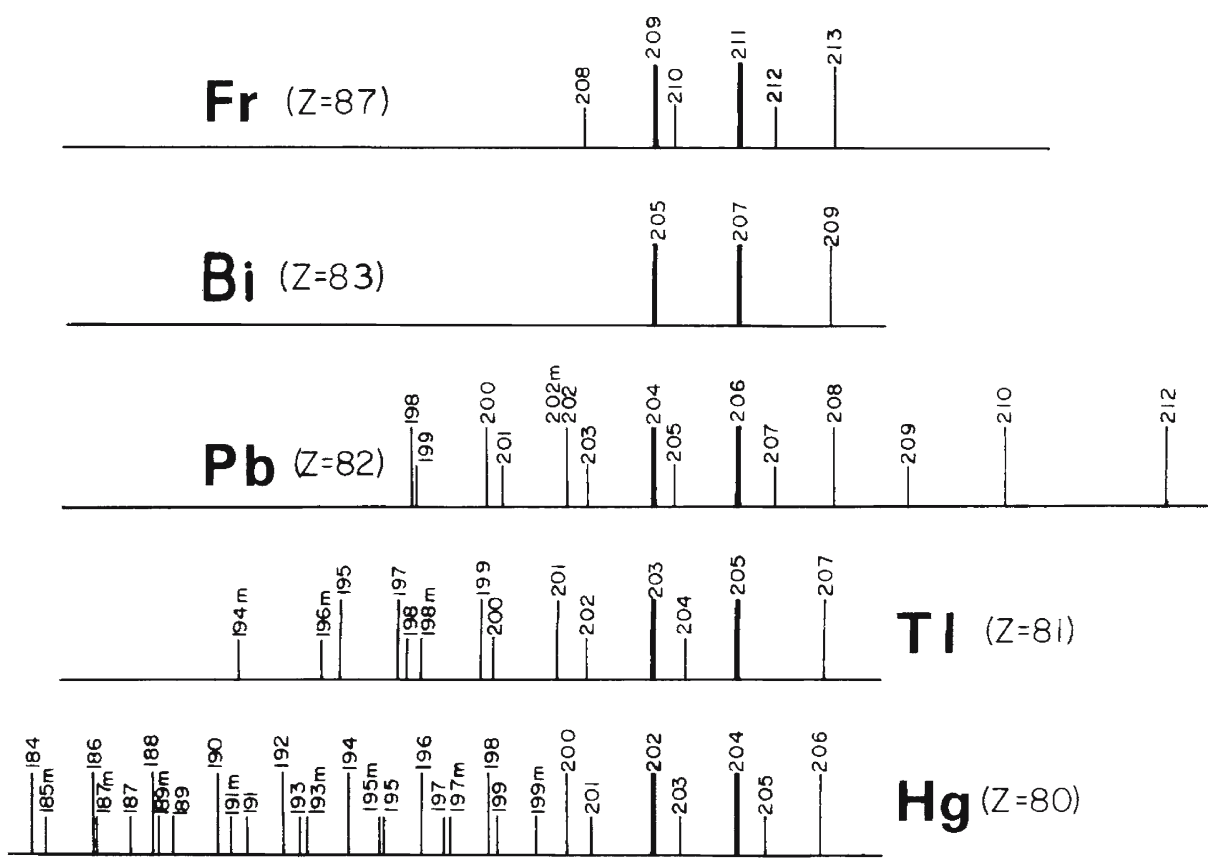

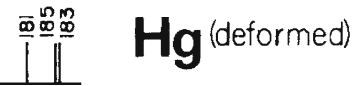

Fig. 43 Isotone shifts in the region of the doubly-magic nucleus, ${ }^{208} \mathrm{~Pb}$ (from [90])

Fig. 44 "Hfs anomalies", or Bohr-Weisskopf effect in cesium radioisopes (from [68], courtesy (CAmerican Physical Society, http://prola.aps.org/ abstact/PR/v105/i2/p590_1)

\begin{tabular}{|cc|cc|c|}
\hline \hline \multicolumn{2}{|c|}{ Isotopes } & \multicolumn{2}{|c|}{ Isotope 1 } & $-\Delta_{12}$ \\
1 & 2 & $\mathrm{I}$ & $\mu\left(\mu_{N}\right)$ & percent \\
\hline${ }^{135} \mathrm{Cs}$ & ${ }^{133} \mathrm{Cs}$ & $7 / 2$ & $2.7290(3)$ & $+0.037 \pm 0.009$ \\
${ }^{137} \mathrm{Cs}$ & ${ }^{133} \mathrm{Cs}$ & $7 / 2$ & $2.8382(3)$ & $+0.009 \pm 0.010$ \\
${ }^{137} \mathrm{Cs}$ & ${ }^{135} \mathrm{Cs}$ & & & $-0.020 \pm 0.009$ \\
${ }^{134} \mathrm{Cs}$ & ${ }^{133} \mathrm{Cs}$ & 4 & $2.9900(9)$ & $+0.169 \pm 0.030$ \\
${ }^{133} \mathrm{Cs}$ & & $7 / 2$ & $2.57893(3)$ & \\
\hline \hline
\end{tabular}

\subsection{Extended nuclear magnetization}

In Section 2.2.2 this effect, additional to the simple magnetic dipole electron-nuclear interaction was introduced. Experimentally, it is revealed by the difference, $\Delta$, between the ratio of the hfs interaction constants, $a$, for two isotopes and the ratio 基 Springer 
Fig. 45 Hartmut Backe. Valentin Fedoseev can be recognized in the background (courtesy H.-J. Kluge)

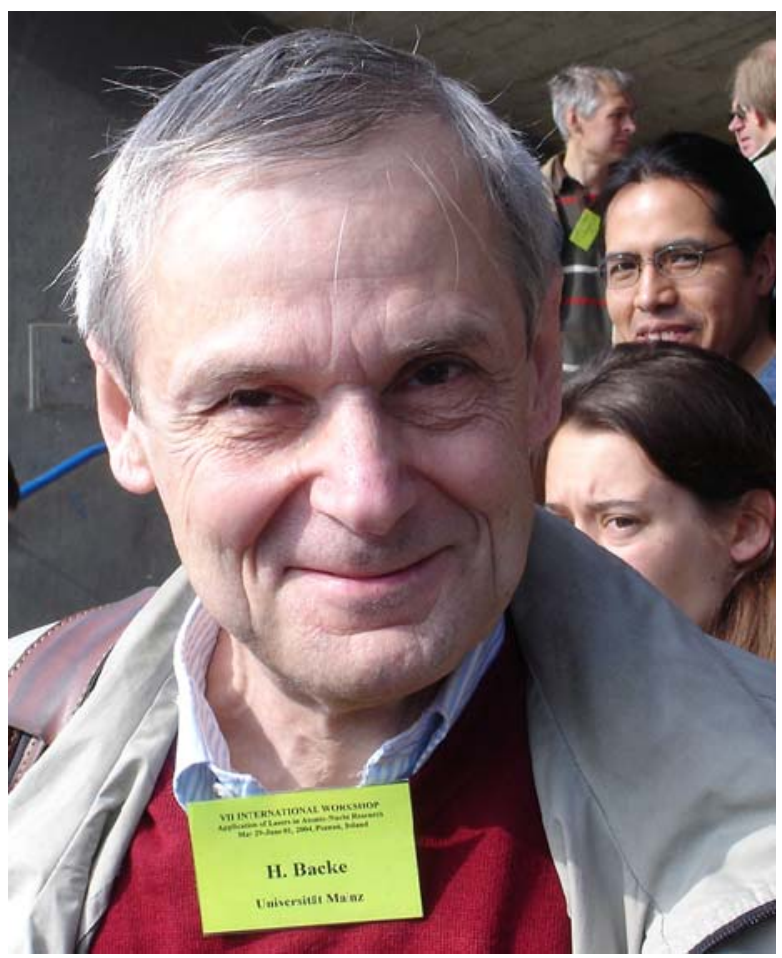

of the nuclear gyromagnetic ratios, $g$. We have $a=a_{\text {point-nucleus }}(1+\varepsilon), \Delta_{12}=\varepsilon_{1}-\varepsilon_{2}$ and take $\left(\frac{a_{1}}{a_{2}}\right)_{\text {point }}=\frac{g_{1}}{g_{2}}$. Then

$$
\frac{a_{1}}{a_{2}} \frac{g_{2}}{g_{1}}-1 \equiv \Delta
$$

For a set of cesium radioisotopes [68] it was found that while the magnetic moments change monotonically for three nuclei with identical spins, $\Delta$ changes signs between successive pairs, Fig. 44 . This would not be the case were $\varepsilon$ simply proportional to the nuclear magnetic moment.

While, qualitatively, a magic neutron number effect could be invoked because of $N=82$ in ${ }^{137} \mathrm{Cs}$, the results required a picture that takes into account details of the nucleon configurations. This motivated a more quantitative approach, which involved nuclear configuration mixing theory [91].

\section{Conclusion}

Working back, magnetic moments, together with the Bohr-Weisskopf effect, can give information on neutron wave functions in nuclei. As suggested, isotope shifts can also provide such insights from systematic studies of relative shifts. Additionally, these 


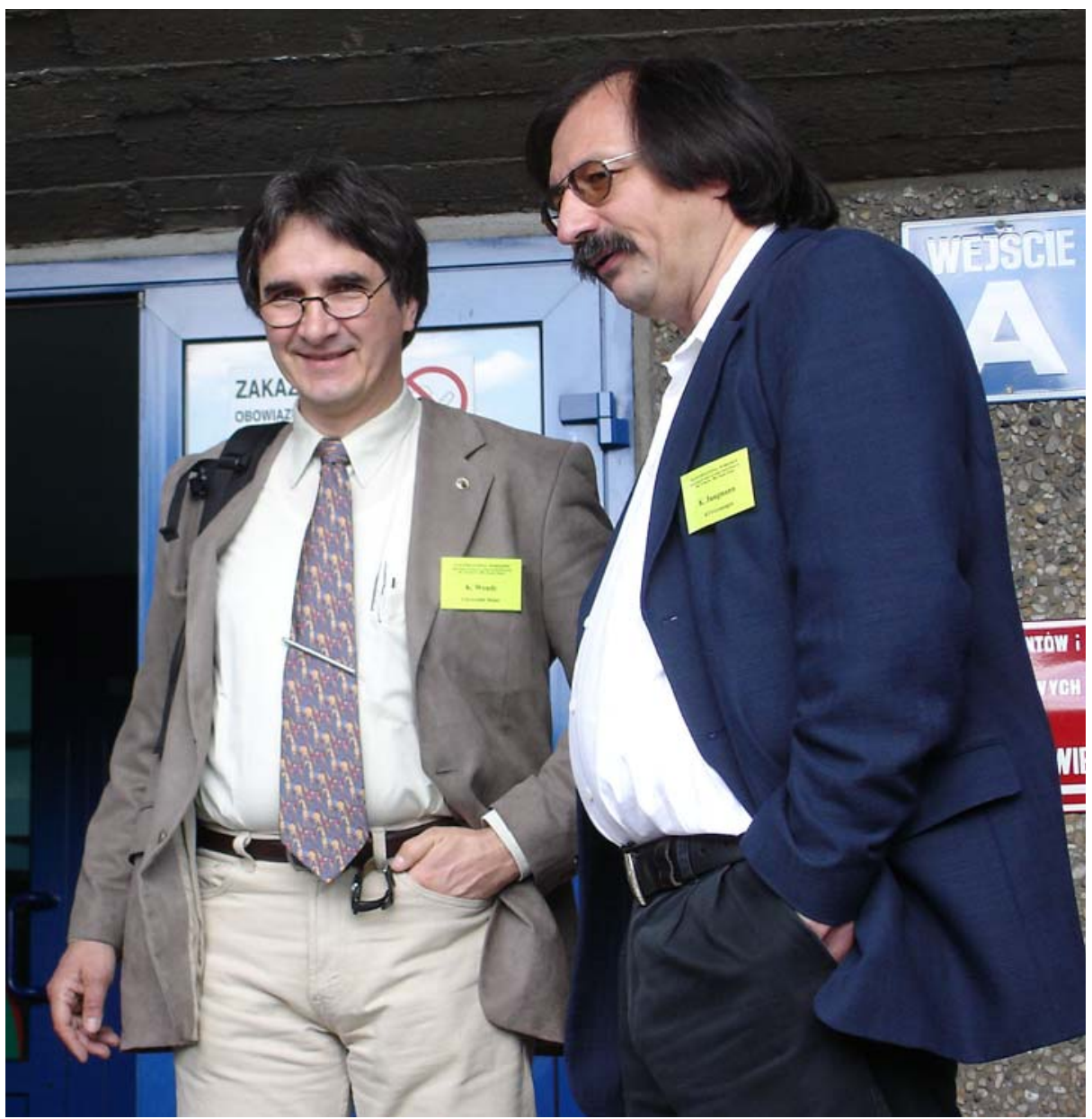

Fig. 46 The two Klaus's: Wendt, left, Jungmann, right (courtesy H.-J. Kluge)

can provide a handle on the neutron-nuclear interaction. Magnetic moments and the Bohr-Weisskopf effect also find importance in extracting quantum-electrodynamic effects from the hfs of atoms highly stripped of electrons. These are studied extensively at GSI, Darmstadt, and are reported at this Workshop by Hartmut Backe (Fig. 45).

Knowledge of neutron wave functions is also required in the interpretation of isotopically-differential parity non-conservation experiments. Such fundamental problems were discussed at the Workshop by Klaus Jungmann, Fig. 46.

Magnetic moments can, in particular nuclei, provide information on pionexchange contributions. And then we can venture far afield in applications, such as the relation of the neutron-rich skin of heavy nuclei and neutron star structure, isotope-shift data for space-time variation of the fine structure constant, and surely a good number of exciting, still undiscovered new endeavors! 


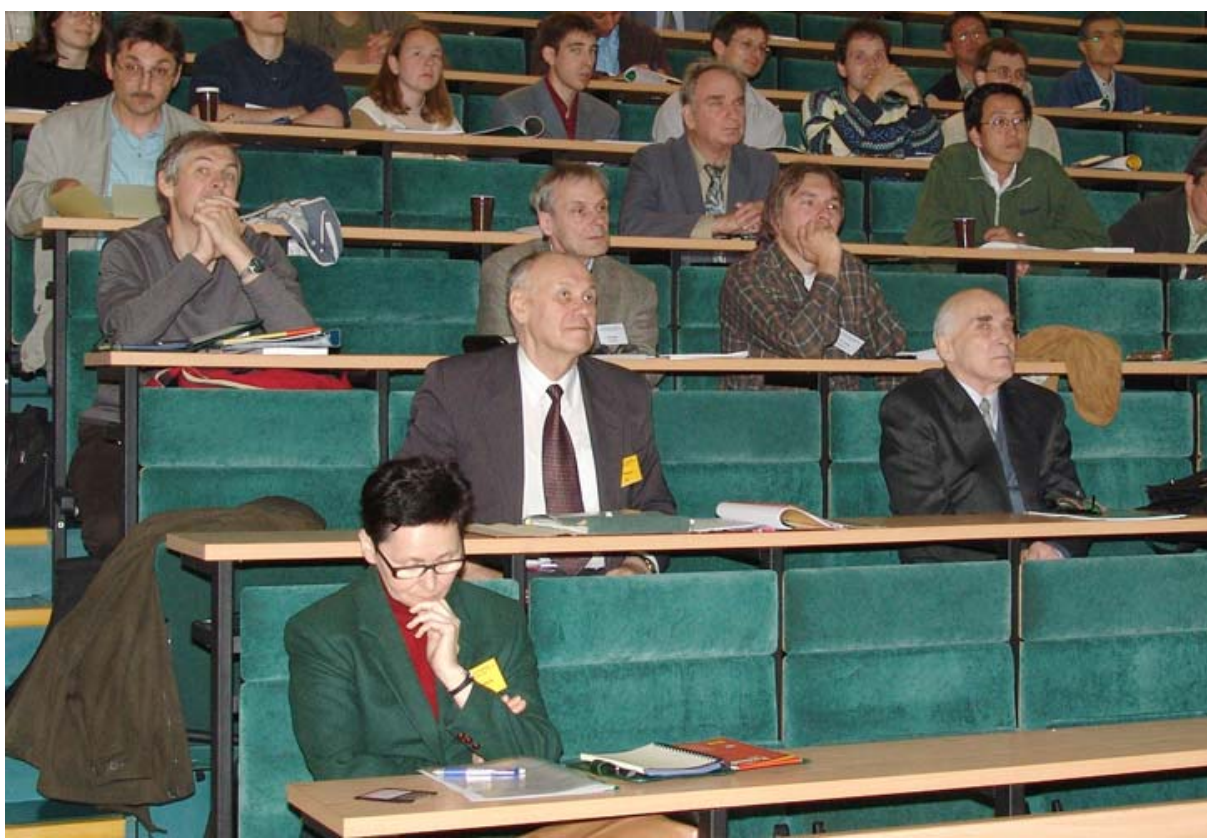

Fig. 47 Krassimira Marinova, moving spirit of the Workshop, first row. Second row, left to right, Boris Markov, Yury Gangsky; third row, Valentin Fedoseev, Hartmut Backe, Werner Lauth; fourth row, Serguei Zemlyanoi, Nikolai Tarantin, Zheng-Tian Lu; fifth row, Dana Borremans, Klaus Blaum, and two seats over to the right, Wilfried Nörteshäuser; Takashi Inamura is seen at upper right hand corner (courtesy R. Jarzebinska)

Acknowledgements I am grateful to the organizers of the Workshop, in particular Jürgen Kluge and Krassimira Marinova, Fig. 47, for the invitation to present at this Workshop this (somewhat biased toward personal experience) sketchy rendition of radioactive atom spectroscopy. I also thank the Physics Faculty of the Adam Mickiewicz University, Poznan, for their hospitality. I appreciate Tak Inamura's enlightening me on the early contributions of Nagaoka to hfs and isotope shift studies. I also want to acknowledge long-term collaborations, and friendly and stimulating interactions with many colleagues, among them Olav Redi, with them much of my work was carried out, Jacques Pinard and H.T. Duong, Laboratoire Aimé Cotton, Jürgen Kluge, Gerhard Huber, Rainer Neugart, Ernst Otten, Mainz and GSI, and many others whose names would fill a good number of pages. They built on the foundations of I.I. Rabi, Hans Kopfermann, Pierre Jacquinot, Jerrold Zacharias, Francis Bitter, Jean Brossel, Alfred Kastler, to name a few, and, in turn, trained a whole new generation of young physicists, many of whom are presenting their contributions at this Workshop.

\section{References}

1. Nierenberg, W.A.: Annu. Rev. Nucl. Sci. 7, 349 (1957)

2. Jacquinot, P., Klapisch, R.: Rep. Prog. Phys. 42, 773 (1979)

3. Bemis Jr., C.E., Carter, H.K.: Lasers in Nuclear Physics. Harwood Academic, Chur (1982)

4. Otten, E.-W.: In Treatise on Heavy-Ion Science, vol. 8, Bromley, D.A. (ed.), Plenum, New York, pp. 517 (1989)

5. Vergnes, M., Goutte, D., Heenen, P.H., Sauvage, J. (eds.): Nuclear shapes and nuclear structure at low excitation energies, Editions Frontières, Gif-sur-Yvette, (1994)

6. Kluge, H.-J., Nörteshäuser, W.: Spectrochim. Acta, Part B: Atom. Spectrosc. 58, 1031 (2003)

7. Billowes, J.: Nucl. Phys., A 752, 309c (2005)

8. Billowes, J.: Hyperfine Interact. 162, 63 (2005) 
9. Rao, G.N. (ed.): Modern Optics, Lasers and Laser Spectroscopy, Hyperfine Interactions, vol. 27 and 38 (1987)

10. Inamura, T.T., Wakasugi, W. (eds.): Lasers in Nuclear Physics, Hyperfine Interactions, vol. 74 (1992)

11. Schweikhard, L., Kluge, H.-J. (eds.): Atomic Physics at Accelerators: Laser Spectroscopy and Applications (APAC99), Hyperfine Interactions, vol. 127 (2000)

12. Blaszczak, Z., Markov, B., Marinova, K. (eds.): Laser 2004: Application of Lasers in Atomic Nuclear Research, ed. Hyperfine Interactions, vol. 162 (2005)

13. Pinard, J., Stroke, H.H.: Adv. Atom. Molec. Opt. Phys. 51, 273 (2005)

14. Michelson, A.A.: Studies in Optics. Phoenix-University of Chicago, Chicago, p.34 (1927)

15. Françon, M.: Optical Interferometry. Academic, New York, 1966

16. Colloques Internationaux du CNRS, No. 161, 1966, Editions du CNRS, Paris, 1967 (references to work by Connes, P., Connes, J., Jacquinot, P., Pinard, J., et al.)

17. de Marcillac, P., Coron, N., Dambier, G., Leblanc, J., Moalic, J.-P.: Nature, 422, 876 (2003)

18. Pauli, W.: Naturwiss. 12, 741 (1924)

19. Back, E., Goudsmit, S.: Z. Phys. 43, 321 (1927)

20. Back, E., Goudsmit, S.: Z. Phys. 47, 174 (1928)

21. Inamura, T.T.: Hyperfine Interact. 127, 31 (2000)

22. Nagaoka, H., Mishima, T.: Proc. Imp. Acad. 2, 249 (1926)

23. Condon, E.U., Shortley, G.H.: Theory of Atomic Spectra. University Press, Cambridge, pp. 420 (1935)

24. Rosenthal, J.E., Breit, G.: Phys. Rev. 41, 459 (1932)

25. Yagoda, H.: Phys. Rev. 38, 2298 (1931)

26. Yagoda, H.: Phys. Rev. 40, 1017 (1932)

27. Cox, R.T., Mcllwraith, C.G., Kurrelmeyer, B.: Proc. Natl. Acad. Sci. U.S.A. 14, 544 (1928)

28. Weisskopf, V.F.: Ann. Phys. (Leipzig) 9, 23 (1931) (Eq. 54)

29. Colgrove, F.D., Franken, P.A., Lewis, R.R., Sands, R.H.: Phys. Rev. Lett. 3, 420 (1959)

30. Bitter, F.: Appl. Opt. 1, 1 (1962)

31. Perey, M.: C.R. Acad. Sci. 208, 97 (1939)

32. Liberman, S., Pinard, J., Duong, H.T., Juncar, P., Vialle, J.-L., Jacquinot, P., Huber, G., Touchard, F., Büttgenbach, S., Pesnelle, A., Thibault, C., Klapisch, R.: C.R. Acad. Ser. B, 286, $253(1978)$

33. Liberman, S., Pinard, J., Duong, H.T., Juncar, P., Pillet, P., Vialle, J.-L., Jacquinot, P., Touchard, F., Büttgenbach, S., Thibault, C., de Saint-Simon, M., Klapisch, R., Pesnelle, A., Huber, G.: Phys. Rev., A 22, 2732 (1980)

34. Goudsmit, S., Bacher, R.F.: Phys. Rev. 34, 1501 (1929)

35. Casimir, H.B.G.: In: Archives du Musée Teyler, Ser. III, VIII, Martinus Nijhoff, The Hague, pp. 201 (1936)

36. Casimir, H.B.G., Karreman, G.: Physica 9, 494 (1942)

37. Jaccarino, V., King, J.G., Satten, R.A., Stroke, H.H.: Phys. Rev. 94, 1798 (1954)

38. de-Shalit, A., Talmi, I.: Nuclear Shell Theory. Academic, New York, pp. 215 (1963)

39. Bohr, A., Weisskopf, V.F.: Phys. Rev. 77, 94 (1950)

40. Kopfermann, H.: Kernmomente, Akademische Verlagsgesellschaft, Leipzig (1940)

41. Kopfermann, H.: Nuclear Moments. Academic, New York, (1958)

42. Cohen, V.W.: Phys. Rev. 46, 713 (1934)

43. Jaccarino, V., Bederson, B., Stroke, H.H.: Phys. Rev. 87, 676 (1952)

44. Rabi, I.I., Zacharias, J.R., Millman, S., Kusch, P.: Phys. Rev. 53, 318(L) (1938)

45. Zacharias, J.R.: Phys. Rev. 61, 270 (1942)

46. Davis Jr., L., Nagle, D.E., Zacharias, J.R.: Phys. Rev. 76, 1068 (1949)

47. Gilbert, D.A., Cohen, V.W.: Phys. Rev. 95, 569 (1955)

48. Lemonick, A., Pipkin, F.M., Hamilton, D.R.: Rev. Sci. Instrum. 26, 1112 (1955)

49. Bitter, F.: Phys. Rev. 76, 833 (1949)

50. Brossel, J., Bitter, F.: Phys. Rev. 86, 308 (1952)

51. Bitter, F.: Selected papers and commentaries, Erber T., Fowler, C.M. (eds.), MIT, Cambridge, Massachusetts (1969)

52. Hirsch, H.R.: PhD Thesis, MIT (1960)

53. Hirsch, H.R.: Opt. J. Soc. Am. 51, 1192 (1961) 
54. Sorokin, P.P., Lankard, J.P.: IBM J. Res. Dev. 10, 162 (1966)

55. Schäfer, F.P., Schmidt, W., Volze, J.: Appl. Phys. Lett. 9, 306 (1966)

56. Schein, M.: Suppl. 1, Helv. Phys. Acta 2, 73 (1929)

57. Kohler, R.H.: PhD Thesis, MIT, (1961)

58. Kohler, R.H.: Phys. Rev. 121, 1104 (1961)

59. Brot, P.C.: J. Phys. Radium 22, 412 (1961)

60. Kastler, A.: J. Phys. Radium 11, 255 (1950)

61. Bitter, F., Brossel, J.: Phys. Rev. 85, 1051 (1952)

62. Mitchell, A.C.G., Zemansky, M.W.: Resonance Radiation and Excited Atoms. University Press, Cambridge (1934)

63. Cagnac, B., Barrat, J.P.: C.R. Acad. Sci. 249, 534 (1959)

64. Cagnac, B.: PhD Thesis, Paris (1960)

65. Cagnac, B.: Ann. Phys. (Paris) 6, 467 (1961)

66. Walter, W.T.: PhD Thesis, MIT (1962)

67. Walter, W.T.: Bull. Am. Phys. Soc. 7, 295 (1962)

68. Stroke, H.H., Jaccarino, V., Edmonds Jr., D.S., Weiss, R.: Phys. Rev. 105, 590 (1957)

69. Besch, H.J., Köpf, U., Otten, E.W.: Phys. Lett., B 25, 120 (1967)

70. Bonn, J., Huber, G., Kluge, H.-J., Köpf, U., Kugler, L., Otten, E.W.: Phys. Lett., B 36, 41 (1971)

71. Bonn, J., Huber, G., Kluge, H.-J., Otten, E.W.: Phys. Lett., B 38, 308 (1972)

72. Ekström, C.: Adv. Quant. Chem. 30, 361 (1998)

73. Huber, G., Touchard, F., Büttgenbach, S., Thibault, C., Klapisch, R., Duong, H.T., Liberman, S., Pinard, J., Vialle, J.L., Juncar, P., Jacquinot, P.: Phys. Rev., C 18, 2342 (1978)

74. Liberman, S., Pinard, J., Duong, H.T., Juncar, P., Vialle, J.-L., Jacquinot, P., Huber, G., Touchard, F., Büttgenbach, S., Pesnelle, A., Thibault, C., Klapisch, R.: CERN Collaboration, C. R. Acad. Ser. B 286, 253 (1978)

75. Thibault, C., Touchard, F., Büttgenbach, S., Klapisch, R., de Saint-Simon, M., Duong, H.T., Jacquinot, P., Juncar, P., Liberman, S., Pillet, P., Pinard, J., Vialle, J.L., Pesnelle, A., Huber, G.: Phys. Rev., C 23, 2720 (1981)

76. Pinard, J., Duong, H.T., Marescaux, D., Stroke, H.H., Redi, O., Gustaffson, M., Nilsson, T., Matsuki, S., Kishimoto, Y., Kominato, K., Ogawa, J., Shibata, M., Tada, M., Persson, J.R., Nojiri, Y., Momota, S., Inamura, T.T., Wakasugi, M., Juncar, P., Murayama, T., Nomura, T., Koizumi, M.: ISOLDE collaboration. Nucl. Phys., A 753, 3 (2005)

77. Kluge, H.-J.: Hyperfine Interactions 37, 347 (1987)

78. Anton, K.-R., Kaufman, S.L., Klempt, W., Moruzzi, G., Neugart, R., Otten, E.W., Schinzler, B.: Phys. Rev. Lett. 40, 642 (1978)

79. Mueller, A.C., Buchinger, F., Klempt, W., Otten, E.W., Neugart, R., Ekström, C., Heinemeier, J.: Nucl. Phys., A 403, 234 (1983)

80. Duong, H.T., Juncar, P., Liberman, S., Mueller, A.C., Neugart, R., Otten, E.W., Peuse, B., Pinard, J., Stroke, H.H., Thibault, C., Touchard, F., Vialle, J.L., Wendt, K., and the ISOLDE Collaboration: Europhys. Lett. 3, 175 (1987)

81. Wallmeroth, K., Bollen, G., Dohn, A., Egelhof, P., Grüner, J., Lindenlauf, F., Krönert, U., Campos, J., Rodriguez-Yunta, B., Borge, M.J.G., Venugopalan, A., Wood, J.L., Moore, R.B., Kluge, H.-J.: Phys. Rev. Lett. 58, 1516 (1987)

82. Lee, J.K.P., Savard, G., Crawford, J.E., Thekkadath, G., Duong, H.T., Pinard, J., Liberman, S., Le Blanc, F., Kilcher, P., Obert, J., Oms, J., Putaux, J.C., Roussière, B., Sauvage, J.: ISOCELE Collaboration. Nucl. Instrum. Methods, B 34, 252 (1988)

83. Alkhazov, G.D., Barzakh, A.E., Berlovich, É.I., Denisov, V.P., Dernyatin, A.G., Ivanov, V.S., Zherikhin, A.N., Kompanets, O.N., Letokhov, V.S., Mishin, V.I., Fedoseev, V.N.: JETP Lett. 37, 274 (1983)

84. Fedoseyev, V.N., Letokhov, V.S., Mishin, V.I., Alkhazov, G.D., Barzakh, A.E., Denisov, V.P., Dernyatin, A.G., Ivanov, V.S.: Opt. Commun. 52, 24 (1984)

85. See, also, Hurst, G.S., Payne, M.G., Kramer, S.D., Young, J.P.: Rev. Mod. Phys. 51, 767 (1979)

86. Sauvage, J., Boos, N., Cabaret, L., Crawford, J.E., Duong, H.T., Genevey, J., Girod, M., Huber, G., Ibrahim, F., Krieg, M., Le Blanc, F., Lee, J.K.P., Libert, J., Lunney, D., Obert, J., Oms, J., Péru, S., Pinard, J., Putaux, J.-C., Roussière, B., Sebastien, V., Verney, D., Zemlyanoi, S., Arianer, J., Barré, N., Ducourtieux, M., Forkel-Wirth, D., Le Scornet, G., Lettry, J., RichardSerre, C., Vernon, C.: Hyperfine Interact. 129, 303 (2000) 
87. Kühl, T., Dabkiewicz, P., Duke, C., Fischer, H., Kluge, H.-J., Kremmling, H., Otten, E.-W.: Phys. Rev. Lett. 39, 180 (1977)

88. Liberman, S., Pinard, J., Duong, H.T., Juncar, P., Vialle, J.L., Pillet, P., Jacquinot, P., Huber, G., Touchard, F., Büttgenbach, S., Thibault, C., Klapisch, R., Pesnelle, A., In: Walther, H., Rothe, K. (eds.): Laser Spectroscopy IV. Springer, Berlin Heidelberg New York, pp. 527 (1979)

89. Stroke, H.H., Proetel, D., Kluge, H.-J.: Phys. Lett., B 82, 204 (1979)

90. Barboza-Flores, M., Redi, O., Stroke, H.H.: Z. Phys., A 321, 85 (1985)

91. Stroke, H.H., Blin-Stoyle, R.J., Jaccarino, V.: Phys. Rev. 123, 1326 (1961) 